\title{
HURWITZ GROUPS OF INTERMEDIATE RANK
}

\section{VSEMIRNOV}

\section{Abstract}

This paper is concerned with $(2,3,7)$-generated linear groups of ranks less than 287. In particular, sixty new values of $n$ are found, such that the groups $\operatorname{SL}_{n}(q)$ are Hurwitz for any prime power $q$. This result provides the next step in deciding which classical groups are Hurwitz.

\section{Introduction}

A group is called $(2,3,7)$-generated if it can be generated by two elements such that they have order 2 and 3, respectively, and their product has order 7 . Finite $(2,3,7)$-generated groups are also known as Hurwitz groups. In other words, the Hurwitz groups are precisely the non-trivial finite homomorphic images of the triangle group

$$
T(2,3,7)=\left\langle X, Y \mid X^{2}=Y^{3}=(X Y)^{7}=1\right\rangle .
$$

The problem of determining which groups are quotients of $T(2,3,7)$ has attracted many researchers. We just mention a recent survey [10], where an overview of the known results is given. Particular attention is paid to the case of classical groups over various rings, especially over finite fields or the ring $\mathbb{Z}$ of integers; see [3, 6, 7]. As is shown in [3], many linear classical groups of rank less than 18 are not Hurwitz. On the other hand, for all sufficiently large ranks, the groups $\operatorname{SL}_{n}(q), \operatorname{Sp}_{2 n}(q), \mathrm{SU}_{2 n}(q)$ and $\Omega_{2 n}^{+}(q)$ for any prime power $q$, and $\mathrm{SU}_{2 n+1}(q)$ and $\Omega_{2 n+1}(q)$ for any odd prime power $q$, are known to be Hurwitz; see $[6,7]$. For example, Lucchini, Tamburini and Wilson proved the following theorem [7, Corollary 1].

THEOREM 1.1 (see [7]). (1) For each prime power $q$ and each integer $n \geqslant 287$, the group $\mathrm{SL}_{n}(q)$ is a Hurwitz group.

(2) For each integer $n \geqslant 287$, the group $\mathrm{SL}_{n}(\mathbb{Z})$ is $(2,3,7)$-generated.

In fact, the above theorem was a consequence of the following - more general - result, which was established in [7, Theorem A]. Given a ring $R$ with identity, let $\mathrm{E}_{n}(R)$ denote the group generated by the set of elementary matrices

$$
\left\{I+r e_{i j}: r \in R, 1 \leqslant i, j \leqslant n, i \neq j\right\} .
$$

Here, $I$ is the identity $n \times n$ matrix and the $e_{i j}$ denote as usual the elements of the standard basis of the matrix algebra $\operatorname{Mat}(n, R)$.

Let $r_{1}, \ldots, r_{m} \in R$. By $R_{r_{1}, \ldots, r_{m}}$, we denote the subring of $R$ (maybe without unity) generated by $r_{1}, \ldots, r_{m}$ (that is, the set of all (finite) $\mathbb{Z}$-linear combinations of monomials $\left.r_{1}^{k_{1}} \cdot \ldots \cdot r_{m}^{k_{m}}, k_{1}+\ldots+k_{m} \geqslant 1\right)$. If $R=R_{r_{1}, \ldots, r_{m}}$, we say that $r_{1}, \ldots, r_{m}$ generate $R$. 
THEOREM 1.2 (see [7]). Let $R$ be a ring that is generated by elements $t_{1}, \ldots, t_{m}$, where $2 t_{1}-t_{1}^{2}$ is a unit of $R$ of finite multiplicative order. Then $\mathrm{E}_{n}(R)$ is $(2,3,7)$-generated for any $n \geqslant 287+84(m-1)$.

In the case of intermediate ranks, however, the problem remains open. The statement of Theorem 1.1 is not the best possible and, as the authors of [7] have already noted in the remark at the end of [7, Section 4], a more careful inspection of their proof shows that $\mathrm{SL}_{n}(q)$ and $\mathrm{SL}_{n}(\mathbb{Z})$ are $(2,3,7)$-generated for any $n$ in the set

$$
S=\{14 m+d: m \geqslant 6, d \in D\} \cup\{42+d: d \in D\},
$$

where

$$
D=\{36,42,57,77,115,135,136,142,144,165,180,187,195,216\} .
$$

There are 93 integers less than 286 in the set $S$. These are:

$78,84,99,119,120,126,134,140,141,148,154,155,157,161,162,168$, $169,175,176,177,178,182,183,184,186,189,190,196,197,199,203,204$, $207,210,211,213,217,218,219,220,222,224,225,226,227,228,229,231$, $232,233,234,237,238,239,240,241,242,245,246,247,248,249,252,253$, $254,255,256,258,259,260,261,262,263,264,266,267,268,269,270,271$, $273,274,275,276,277,278,279,280,281,282,283,284,285$.

The aim of the present paper is to investigate groups of other intermediate ranks. Our main result is the following theorem.

THEOREM 1.3. Let $R$ be a ring that is generated by an element $t$. Assume that 1 belongs to the subring of $R$ generated by $2 t-t^{2}$. Let $n$ be in the set

$$
\begin{aligned}
& \{49,57,63,64,70,77,85,91,92,93,98,100,105,106,108,112,113, \\
& 114,121,127,128,129,133,135,136,142,147,149,150,156,163,164, \\
& 165,170,171,172,180,185,191,192,193,198,200,201,205,206,208, \\
& 212,214,216,221,235,236,243,244,250,257,265,272,286\} .
\end{aligned}
$$

Then $\mathrm{E}_{n}(R)$ is $(2,3,7)$-generated. In particular, the groups $\mathrm{SL}_{n}(\mathbb{Z})$ and $\mathrm{SL}_{n}(q)$ for any prime power $q$ are $(2,3,7)$-generated.

Using a slightly different technique, which goes back to [7], M. C. Tamburini independently obtained a special case of Theorem 1.3 for $n=49$, in work that is as yet unpublished.

Another purpose of this paper is to provide general results about new ways of building linear representations of $T(2,3,7)$ from the known ones. For this reason, we state some auxiliary lemmas in a slightly more general form than we actually need here. They will be used in a future research on low-rank Hurwitz groups.

\section{Obtaining new representations via handles}

Let $R$ be a commutative ring with unity. We are mostly interested in two cases, namely, $R=\mathbb{Z}$ and $R=\mathbb{F}_{q}$, a finite field with $q$ elements. Let $\Sigma$, where $|\Sigma|=n$, be the canonical basis for the free $R$-module $\langle\Sigma\rangle=R^{n}$ consisting of row vectors of size $n$. In what follows, 
we consider the action of $\operatorname{Sym}(n)$ on $\Sigma$ and $\mathrm{GL}_{n}(R)$ on $\langle\Sigma\rangle$ on the right. We will identify $\operatorname{Sym}(n)$ with the subgroup of $\mathrm{GL}_{n}(R)$ consisting of permutational matrices.

A very efficient tool for building new permutational representations of $T(2,3,7)$ using coset diagrams is due to Higman. Further developments of these ideas can be found in the papers by Conder [1] and Stothers [9]. They used different language, but the terminology introduced by Conder is now common. For this reason, we refer to [1], where the notion of a handle appeared for the first time.

Definition 2.1. Let $\psi: T(2,3,7) \longrightarrow \operatorname{Sym}(n)$ be a permutational representation of the group $T(2,3,7)$, and let $i \in\{1,2,3\}$. An ordered pair $\left(a_{1}, a_{2}\right)$, where $a_{1}, a_{2} \in \Sigma, a_{1} \neq a_{2}$, is called an $i$-handle for $\psi$ if:

(i) $\psi(X)$ fixes both $a_{1}$ and $a_{2}$;

(ii) $a_{1}(\psi(X) \psi(Y))^{i}=a_{2}$.

We write $\left(a_{1}, a_{2}\right)_{i}$ to indicate that the pair $\left(a_{1}, a_{2}\right)$ is an $i$-handle.

The key step was the following result.

Lemma 2.2 (see [1]). Suppose that $|\Sigma|=n$ and $\left|\Sigma^{\prime}\right|=n^{\prime}$, and that $\Sigma$ and $\Sigma^{\prime}$ are disjoint. Let

$$
\psi: T(2,3,7) \longrightarrow \operatorname{Sym}(n) \text { and } \psi^{\prime}: T(2,3,7) \longrightarrow \operatorname{Sym}\left(n^{\prime}\right)
$$

be two transitive permutational representations of $T(2,3,7)$. Assume further that $\left(a_{1}, a_{2}\right)_{i}$ and $\left(a_{1}^{\prime}, a_{2}^{\prime}\right)_{i}$, where $a_{1}, a_{2} \in \Sigma$ and $a_{1}^{\prime}, a_{2}^{\prime} \in \Sigma^{\prime}$, are $i$-handles for $\psi$ and $\psi^{\prime}$, respectively. Then, putting

$$
\tilde{\psi}(X)=\psi(X) \psi^{\prime}(X)\left(a_{1} a_{1}^{\prime}\right)\left(a_{2} a_{2}^{\prime}\right) \text { and } \tilde{\psi}(Y)=\psi(Y) \psi^{\prime}(Y),
$$

we define a transitive representation $\tilde{\psi}: T(2,3,7) \longrightarrow \operatorname{Sym}\left(n+n^{\prime}\right)$.

Remark 2.3. More precisely, Conder [1] also considered representations of the group

$$
T^{*}(2,3,7)=\left\langle X, Y, S \mid X^{2}=Y^{3}=(X Y)^{7}=S^{2}=(X S)^{2}=(Y S)^{2}=1\right\rangle,
$$

which contains $T(2,3,7)$ as a subgroup of index 2 . For this reason, he used representations whose coset diagrams have a vertical axis of symmetry, and imposed a certain symmetry condition in the definition of a handle. However, this symmetry is irrelevant to representations of $T(2,3,7)$, and an analysis of Conder's proof shows that Lemma 2.2 remains valid if handles are defined as above. See also an alternative approach in [9].

Later, Lucchini, Tamburini and Wilson $[6,7]$ extended the notion of a 1-handle to the case of linear representations. We modify their definition slightly to include a more general case.

DEFinition 2.4. Let $\psi: T(2,3,7) \longrightarrow \mathrm{GL}_{n}(R)$ be a representation of the group $T(2,3,7)$. An ordered pair $\left(a_{1}, a_{2}\right)$, where $a_{1}, a_{2} \in \Sigma, a_{1} \neq a_{2}$, is called a handle for $\psi$ if

(i) $\psi(X)$ induces the identity on $\left\langle a_{1}, a_{2}\right\rangle$ and fixes $\left\langle\Sigma \backslash\left\{a_{1}, a_{2}\right\}\right\rangle$;

(ii) $a_{1} \psi(Y)=a_{2}$ and

$$
\left\langle\Sigma \backslash\left\{a_{1}\right\}\right\rangle \psi(Y) \subseteq\left\langle\Sigma \backslash\left\{a_{2}\right\}\right\rangle .
$$

REMARK 2.5. Instead of condition (ii), a stronger condition was used in $[6,7]$, namely that (ii') $\psi(Y)$ acts as $\left(a_{1}, a_{2}, a_{3}\right)$ for some $a_{3} \in \Sigma$ and fixes $\left\langle\Sigma \backslash\left\{a_{1}, a_{2}, a_{3}\right\}\right\rangle$. 
Clearly, any 1-handle in the sense of Definition 2.1 is also a handle in the sense of Definition 2.4.

The following lemma describes how to build new representations using handles. In contrast to [6, Lemma 1, case (1)], where two handles are required, we need only one.

LemMA 2.6. Let $\psi: T(2,3,7) \longrightarrow \mathrm{GL}_{n}(R)$ be a representation of the group $T(2,3,7)$, and let $\left(a_{1}, a_{2}\right)$ be a handle for $\psi$. Assume that $b_{1}, b_{2} \in\left\langle\Sigma \backslash\left\{a_{1}, a_{2}\right\}\right\rangle$, and that the following properties are satisfied:

(i) $\psi(X)$ fixes $b_{1}$ and $b_{2}$;

(ii) $b_{1} \psi(Y)=b_{2}$.

Let $U \in \mathrm{GL}_{n}(R)$ be the matrix that induces the identity on $\left\langle\Sigma \backslash\left\{a_{1}, a_{2}\right\}\right\rangle$ and acts on $a_{1}$ and $a_{2}$ as follows:

$$
a_{1} U=-a_{1}+b_{1} ; \quad a_{2} U=-a_{2}+b_{2} .
$$

Then $U$ and $\psi(X)$ commute, $U$ and $U \psi(X)$ are involutions, and $U \psi(X) \psi(Y)$ is conjugate to $\psi(X) \psi(Y)$. In particular, we can define a new representation $\hat{\psi}: T(2,3,7) \longrightarrow \mathrm{GL}_{n}(R)$ by setting $\hat{\psi}(X)=U \psi(X)$ and $\hat{\psi}(Y)=\psi(Y)$.

Proof. Clearly, $U$ is an involution. Now, note that $U \psi(X)$ and $\psi(X) U$ act on $\left\langle\Sigma \backslash\left\{a_{1}, a_{2}\right\}\right\rangle$ in the same way as $\psi(X)$ does. In addition, for $i=1,2$ :

$$
a_{i} U \psi(X)=-a_{i}+b_{i} ; \quad a_{i} \psi(X) U=-a_{i}+b_{i} .
$$

Therefore, $U \psi(X)=\psi(X) U$ and $(U \psi(X))^{2}=U^{2}(\psi(X))^{2}=1$.

Next, for any $v \in\left\langle\Sigma \backslash\left\{a_{1}, a_{2}\right\}\right\rangle$ we have $v U \psi(X) \psi(Y)=v \psi(X) \psi(Y)$ and

$$
\begin{aligned}
v \psi(X) \psi(Y) & \in\left\langle\Sigma \backslash\left\{a_{1}, a_{2}\right\}\right\rangle \psi(X) \psi(Y) \subseteq\left\langle\Sigma \backslash\left\{a_{1}, a_{2}\right\}\right\rangle \psi(Y) \\
& \subseteq\left\langle\Sigma \backslash\left\{a_{1}\right\}\right\rangle \psi(Y) \subseteq\left\langle\Sigma \backslash\left\{a_{2}\right\}\right\rangle,
\end{aligned}
$$

whereas

$$
\begin{gathered}
U \psi(X) \psi(Y): a_{1} \mapsto-a_{2}+b_{2} \mapsto a_{2} \psi(Y) ; \\
\psi(X) \psi(Y): a_{1} \mapsto a_{2} \mapsto a_{2} \psi(Y) .
\end{gathered}
$$

Let $\Sigma^{\prime}$ be the basis of $R^{n}$ obtained from $\Sigma$ by substituting $-a_{2}+b_{2}$ for $a_{2}$. By (2), we have $a_{2} \psi(Y) \in\left\langle\Sigma \backslash\left\{a_{2}\right\}\right\rangle$. This inclusion and (3)-(5) imply that the matrix of $U \psi(X) \psi(Y)$ with respect to the basis $\Sigma^{\prime}$ coincides with the matrix of $\psi(X) \psi(Y)$ with respect to $\Sigma$. This completes the proof.

REMARK 2.7. If $b_{1}=b_{2} \neq 0$, then assumptions (i) and (ii) of Lemma 2.6 imply that the subspace $\left\langle b_{1}\right\rangle$ is $\langle\psi(X), \psi(Y)\rangle$-invariant; hence it is $\langle\tilde{\psi}(X), \tilde{\psi}(Y)\rangle$-invariant. Since we are mostly interested in irreducible representations, we will consider only the case when $b_{1} \neq b_{2}$.

REMARK 2.8. A special case of Lemma 2.6, namely when $b_{1}=t c_{1}$ and $b_{2}=t c_{2}$ for some handle $\left(c_{1}, c_{2}\right)$ with $c_{1}, c_{2} \in \Sigma$ and for some $t \in R$, appears in $[2,6,7]$.

The following lemma gives us some useful information about the behaviour of the commutator

$$
[\tilde{\psi}(X), \tilde{\psi}(Y)]=\tilde{\psi}(X)^{-1} \tilde{\psi}(Y)^{-1} \tilde{\psi}(X) \tilde{\psi}(Y) .
$$


Lemma 2.9. Under the assumptions of Lemma 2.6, set $\tilde{C}=[\tilde{\psi}(X), \tilde{\psi}(Y)]$ and $C=[\psi(X), \psi(Y)]$. Then the following statements hold.

(i) $a_{2}$ is fixed by $C$ and $\tilde{C}$.

(ii) Set $a_{3}:=a_{2} \psi(Y), a_{4}:=a_{3} \psi(X), b_{3}:=b_{1} \psi\left(Y^{-1}\right)=b_{2} \psi(Y)$ and $b_{5}:=b_{3} \psi(X Y)$ $=b_{1} \psi\left(Y^{-1} X Y\right)$. Suppose further that

$$
a_{3}, a_{4} \in \Sigma \backslash\left\{a_{1}, a_{2}\right\}
$$

and

$$
\begin{gathered}
\left\langle\Sigma \backslash\left\{a_{1}, a_{2}, a_{3}\right\}\right\rangle \text { is fixed by } \psi(Y) ; \\
\left\langle\Sigma \backslash\left\{a_{1}, a_{2}, a_{3}, a_{4}\right\}\right\rangle \psi(X) \subseteq\left\langle\Sigma \backslash\left\{a_{1}, a_{2}, a_{3}\right\}\right\rangle .
\end{gathered}
$$

Then

$$
b_{3} \in\left\langle\Sigma \backslash\left\{a_{1}, a_{2}, a_{3}\right\}\right\rangle .
$$

Finally, under the above assumptions we have $v C=v \tilde{C}$ for any $v \in \Sigma \backslash\left\{a_{1}, a_{4}\right\}$, whereas

$$
\begin{aligned}
& a_{1} C=a_{4} \psi(Y) \\
& a_{4} C=a_{3} \\
& a_{1} \tilde{C}=-a_{4} \psi(Y)+b_{1} \psi\left(Y^{-1} X Y\right)=-a_{1} C+b_{5} \\
& a_{4} \tilde{C}=-a_{3}+b_{2} \psi(Y)=-a_{4} C+b_{3} .
\end{aligned}
$$

Proof. (i) Recall that $\psi\left(X^{-1}\right)=\psi(X), U^{-1}=U$ and the matrices $U$ and $\psi(X)$ commute. Thus $\tilde{C}=\psi(X) U \psi\left(Y^{-1}\right) U \psi(X) \psi(Y)$. Now we have

$$
\begin{aligned}
& a_{2} \stackrel{\psi(X)}{\longmapsto} a_{2} \stackrel{\psi\left(Y^{-1}\right)}{\longmapsto} a_{1} \stackrel{\psi(X)}{\longmapsto} a_{1} \stackrel{\psi(Y)}{\longmapsto} a_{2}, \\
& a_{2} \stackrel{\psi(X)}{\longmapsto} a_{2} \stackrel{U}{\longmapsto}-a_{2}+b_{2} \stackrel{\psi\left(Y^{-1}\right)}{\longmapsto}-a_{1}+b_{1} \stackrel{U}{\longmapsto} a_{1} \stackrel{\psi(X)}{\longmapsto} a_{1} \stackrel{\psi(Y)}{\longmapsto} a_{2},
\end{aligned}
$$

which proves part (i).

(ii) To prove inclusion (9), recall that $a_{1}, a_{2}, a_{3} \in \Sigma$ and they are pairwise distinct. Write

$$
b_{3}=\alpha_{1} a_{1}+\alpha_{2} a_{2}+\alpha_{3} a_{3}+w,
$$

where $w \in\left\langle\Sigma \backslash\left\{a_{1}, a_{2}, a_{3}\right\}\right\rangle$. By the definition of $a_{3}$ and $b_{3}$, Definition 2.4 and assumption (7), we have

$$
\begin{aligned}
& b_{1}=b_{3} \psi(Y)=\alpha_{3} a_{1}+\alpha_{1} a_{2}+\alpha_{2} a_{3}+w \psi(Y), \\
& b_{2}=b_{3} \psi\left(Y^{2}\right)=\alpha_{2} a_{1}+\alpha_{3} a_{2}+\alpha_{1} a_{3}+w \psi\left(Y^{2}\right),
\end{aligned}
$$

where $w \psi(Y)$ and $w \psi\left(Y^{2}\right)$ are in $\left\langle\Sigma \backslash\left\{a_{1}, a_{2}, a_{3}\right\}\right\rangle$. Since $b_{1}, b_{2} \in\left\langle\Sigma \backslash\left\{a_{1}, a_{2}\right\}\right\rangle$ by the assumptions of Lemma 2.6, we conclude that $\alpha_{1}=\alpha_{2}=\alpha_{3}=0$; that is, inclusion (9) holds.

Now we are ready to prove the main claim of statement (ii). The case $v=a_{2}$ has already been settled in part (i). Take $v \in \Sigma \backslash\left\{a_{1}, a_{2}, a_{4}\right\}$. First, we show that

$$
v \psi(X) \in\left\langle\Sigma \backslash\left\{a_{1}, a_{2}, a_{3}\right\}\right\rangle,
$$

and, in particular, that

$$
v \psi(X) \in\left\langle\Sigma \backslash\left\{a_{1}, a_{2}\right\}\right\rangle .
$$


For $v \neq a_{3}$, both inclusions are obvious by statement (8). If $v=a_{3}$, then $v \psi(X)=a_{4}$. Therefore, (11) follows from (6). Since $\psi(X Y)$ has order 7, we have $a_{4}=a_{3} \psi(X) \neq a_{3}$, which - together with (6) - implies that inclusion (10) holds in this case, too.

By inclusion (11),

$$
v \psi(X) U=v \psi(X) \quad \text { and } \quad v \psi(X) U \psi\left(Y^{-1}\right)=v \psi\left(X Y^{-1}\right)
$$

for any $v \in \Sigma \backslash\left\{a_{1}, a_{2}, a_{4}\right\}$, while (10) and (7) imply that

$$
v \psi\left(X Y^{-1}\right) \in\left\langle\Sigma \backslash\left\{a_{1}, a_{2}, a_{3}\right\}\right\rangle \subseteq\left\langle\Sigma \backslash\left\{a_{1}, a_{2}\right\}\right\rangle .
$$

Therefore,

$$
v \psi(X) U \psi\left(Y^{-1}\right) U=v \psi\left(X Y^{-1}\right)
$$

and

$$
v \tilde{C}=v \psi(X) U \psi\left(Y^{-1}\right) U \psi(X Y)=v \psi\left(X Y^{-1} X Y\right)=v \psi\left(X^{-1} Y^{-1} X Y\right)=v C .
$$

Finally, we have

$$
\begin{aligned}
a_{1} & \stackrel{\psi(X)}{\longmapsto} a_{1} \stackrel{\psi\left(Y^{-1}\right)}{\longmapsto} a_{3} \stackrel{\psi(X)}{\longmapsto} a_{4} \stackrel{\psi(Y)}{\longmapsto} a_{4} \psi(Y) ; \\
a_{4} & \stackrel{\psi(X)}{\longmapsto} a_{3} \stackrel{\psi\left(Y^{-1}\right)}{\longmapsto} a_{2} \stackrel{\psi(X)}{\longmapsto} a_{2} \stackrel{\psi(Y)}{\longmapsto} a_{3} ; \\
a_{4} & \stackrel{\psi(X)}{\longmapsto} a_{3} \stackrel{U}{\longmapsto} a_{3} \stackrel{\psi\left(Y^{-1}\right)}{\longmapsto} a_{2} \stackrel{U}{\longmapsto}-a_{2}+b_{2} \stackrel{\psi(X)}{\longmapsto}-a_{2}+b_{2} \stackrel{\psi}{\longmapsto}-a_{3}+b_{2} \psi(Y) ; \\
a_{1} & \stackrel{\psi(X)}{\longmapsto} a_{1} \stackrel{\leftrightarrow}{\longmapsto}-a_{1}+b_{1} \stackrel{\psi\left(Y^{-1}\right)}{\longmapsto}-a_{3}+b_{1} \psi\left(Y^{-1}\right) \stackrel{U}{\longmapsto}-a_{3}+b_{1} \psi\left(Y^{-1}\right) \\
& \stackrel{\psi(X)}{\longmapsto}-a_{4}+b_{1} \psi\left(Y^{-1} X\right) \stackrel{\psi(Y)}{\longmapsto}-a_{4} \psi(Y)+b_{1} \psi\left(Y^{-1} X Y\right) ;
\end{aligned}
$$

for the second occurrence of $U$ in the last chain, we use (9). The proof is complete.

LEMMA 2.10. Under the assumptions and notations of Lemma 2.9, suppose further that:

(i) $\left\{a_{1}, a_{4}\right\} \subseteq \Delta \subseteq \Sigma$, where both $\langle\Delta\rangle$ and $\langle\Sigma \backslash \Delta\rangle$ are invariant under $C$;

(ii) $|\Delta|=s$ and $C$ acts on $\Delta$ as a cycle of the following shape: $(\underbrace{a_{1}, \ldots,}_{k} \underbrace{a_{4}, \ldots}_{s-k})$;
(iii) $b_{3}$ and $b_{5}$ are in $\langle\Sigma \backslash \Delta\rangle$;

(iv) both vectors $b_{5} C^{k}-b_{3}$ and $b_{3} C^{s-k}-b_{5}$ are annihilated by the matrix $f(C)$, where the polynomial $f$ is given by

$$
f(z)=\left(z^{r}-1\right) /\left(z^{\operatorname{gcd}(r, s)}-1\right) \quad \text { for some } r .
$$

Then $\tilde{C}^{r s}=C^{r s}$.

Proof. By Lemma 2.9, $\tilde{C}$ and $C$ act in the same way on $\langle\Sigma \backslash \Delta\rangle$. Since $\langle\Sigma \backslash \Delta\rangle$ is invariant under $C$, we find that

$$
v \tilde{C}^{\ell}=v C^{\ell} \text { for any } v \in\langle\Sigma \backslash \Delta\rangle \text { and for any } \ell \geqslant 0 .
$$

By (ii), $\Delta=\left\{a_{1} C^{-s+k+1}, \ldots, a_{1}, \ldots, a_{1} C^{k}\right\}$, and $a_{1} C^{k}=a_{4}$. Using Lemma 2.9, we obtain $a_{1} \tilde{C}=-a_{1} C+b_{5}, a_{1} C^{k} \tilde{C}=-a_{1} C^{k+1}+b_{3}$, and $a_{1} C^{l} \tilde{C}=a_{1} C^{l+1}$ if $l \neq \equiv 0, k$ $\bmod s$. Therefore, $a_{1} C^{l} \tilde{C}^{i}=a_{1} C^{l+i}$, provided that $i=0$ or $i \geqslant 1$ and the sequence $l, l+1, \ldots, l+i-1$ contains no number congruent to 0 or $k(\bmod s)$. Taken together 
with equation (12) and assumption (iii), this implies that, for $j=0, \ldots, s-k-1$, we have

$$
\begin{aligned}
a_{1} C^{-j} \tilde{C}^{s} & =a_{1} C^{-j} \tilde{C}^{j} \tilde{C}^{s-j}=a_{1} \tilde{C}^{s-j} \\
& =\left(-a_{1} C+b_{5}\right) \tilde{C}^{s-j-1}=-a_{1} C \tilde{C}^{k-1} \tilde{C}^{s-j-k}+b_{5} C^{s-j-1} \\
& =-a_{1} C^{k} \tilde{C}^{s-j-k}+b_{5} C^{s-j-1}=\left(a_{1} C^{k+1}-b_{3}\right) \tilde{C}^{s-j-k-1}+b_{5} C^{s-j-1} \\
& =a_{1} C^{s-j}-b_{3} C^{s-j-k-1}+b_{5} C^{s-j-1} \\
& =a_{1} C^{-j}+\left(-b_{3}+b_{5} C^{k}\right) C^{s-j-k-1},
\end{aligned}
$$

while for $j=1, \ldots, k$, we have

$$
\begin{aligned}
a_{1} C^{j} \tilde{C}^{s} & =a_{1} C^{j} \tilde{C}^{k-j} \tilde{C}^{s-k+j}=a_{1} C^{k} \tilde{C}^{s-k+j} \\
& =\left(-a_{1} C^{k+1}+b_{3}\right) \tilde{C}^{s-k+j-1}=-a_{1} C^{k+1} \tilde{C}^{s-k-1} \tilde{C}^{j}+b_{3} C^{s-k+j-1} \\
& =-a_{1} C^{s} \tilde{C}^{j}+b_{3} C^{s-k+j-1}=-a_{1} \tilde{C}^{j}+b_{3} C^{s-k+j-1} \\
& =\left(a_{1} C-b_{5}\right) \tilde{C}^{j-1}+b_{3} C^{s-k+j-1}=a_{1} C \tilde{C}^{j-1}-b_{5} C^{j-1}+b_{3} C^{s-k+j-1} \\
& =a_{1} C^{j}+\left(-b_{5}+b_{3} C^{s-k}\right) C^{j-1} .
\end{aligned}
$$

In addition, assumptions (i) and (iii) imply that both $-b_{3}+b_{5} C^{k}$ and $-b_{5}+b_{3} C^{s-k}$ are in $\langle\Sigma \backslash \Delta\rangle$. Therefore, for any $j$ we have

$$
a_{1} C^{j} \tilde{C}^{s}=a_{1} C^{j}+u_{j} C^{\alpha_{j}}
$$

where $\alpha_{j}$ is a non-negative integer, $u_{j}$ is annihilated by $f(C)$, and

$$
u_{j} \in\langle\Sigma \backslash \Delta\rangle \text {. }
$$

Let $\Phi_{d}(z)$ denote, as usual, the $d$ th cyclotomic polynomial. Over any field of characteristic 0 , we have

$$
\begin{aligned}
z^{r s}-1 & =\prod_{d \mid r s} \Phi_{d}(z) \\
& =\prod_{d \mid s} \Phi_{d}(z) \prod_{d \mid r} \Phi_{d}(z)\left(\prod_{d \mid \operatorname{gcd}(r, s)} \Phi_{d}(z)\right)^{-1} h(z) \\
& =\left(z^{s}-1\right)\left(z^{r}-1\right)\left(z^{\operatorname{gcd}(r, s)}-1\right)^{-1} h(z) \\
& =\left(z^{s}-1\right) f(z) h(z),
\end{aligned}
$$

All the polynomials $\Phi_{d}$ are polynomials with integer coefficients, and thus $h$ is too. Therefore, the decomposition

$$
z^{r s}-1=\left(z^{s}-1\right) f(z) h(z)
$$

holds over every ring with unity. Now, using (13), (14), assumptions (i) and (iii), and the fact that $C$ and $\tilde{C}$ act in the same way on $\langle\Sigma \backslash \Delta\rangle$, we deduce that

$$
\begin{aligned}
a_{1} C^{j}\left(\tilde{C}^{r s}-I\right) & =a_{1} C^{j}\left(\tilde{C}^{s}-I\right) f(\tilde{C}) h(\tilde{C}) \\
& =u_{j} C^{\alpha_{j}} f(\tilde{C}) h(\tilde{C}) \\
& =u_{j} C^{\alpha_{j}} f(C) h(C) \\
& =u_{j} f(C) h(C) C^{\alpha_{j}} \\
& =0 .
\end{aligned}
$$

Hence, $\tilde{C}^{r s}$ induces the identity on $\langle\Delta\rangle$. Clearly, $C^{r s}$ does the same. Therefore, $\tilde{C}^{r s}=C^{r s}$ on $R^{n}$. 
LEMMA 2.11. Conditions (iii) and (iv) of Lemma 2.10 are satisfied, for example, if $b_{3}=t c_{3}$, $b_{5}=t c_{5}$ with $\left\{c_{3}, c_{5}\right\} \subseteq \Delta^{\prime} \subseteq(\Sigma \backslash \Delta), C$ acts on $\Delta^{\prime}$ as a cycle of length $r=\left|\Delta^{\prime}\right|$, and one of the following conditions holds:

(i) $r=s$ and $c_{5} C^{k}=c_{3}$, where $k$ is the same as in Lemma 2.10;

(ii) $\operatorname{gcd}(r, s)=1$.

Proof. Clearly, in the first case, $f(z)=1$ and $-b_{3}+b_{5} C^{k}=-b_{5}+b_{3} C^{s-k}=0$. In the second case, $f(z)=1+z+\ldots+z^{r-1}$ Hence

$$
\begin{aligned}
b_{3} f(C) & =\sum_{i=0}^{r-1} b_{3} C^{i}=t \sum_{v \in \Delta^{\prime}} v=\sum_{i=0}^{r-1} b_{5} C^{i+k} \\
& =b_{5} C^{k} f(C) .
\end{aligned}
$$

In a similar way, $b_{3} C^{s-k} f(C)=b_{5} f(C)$.

\section{Some generation lemmas}

In this section we prove several auxiliary results about generating sets for the groups $\operatorname{Alt}(n)$ and $\mathrm{E}_{n}(R)$. The technique is due to A. Lucchini, M. C. Tamburini and J. S. Wilson; see, for example, $[6,7]$. Most of the statements in this section may be regarded as non-trivial refinements of similar results in [6] and [7].

LeMma 3.1. Let $H$ be a subgroup of $\operatorname{Alt}(k) \times \operatorname{Alt}(m)$, where $k>\max \{m, 4\}$. Let $\pi_{i}$, $i=1,2$, be the natural projections from $\operatorname{Alt}(k) \times \operatorname{Alt}(m)$ to $\operatorname{Alt}(k)$ and $\operatorname{Alt}(m)$, respectively. Assume that $\pi_{1}(H)=\operatorname{Alt}(k)$. Then $H=\operatorname{Alt}(k) \times B$, where $B=\pi_{2}(H)$. In particular, $\operatorname{Alt}(k) \times\langle 1\rangle \leqslant H$.

Proof. We have ker $\pi_{2} \cap H \unlhd H$. Consequently, $\pi_{1}\left(\operatorname{ker} \pi_{2} \cap H\right) \unlhd \pi_{1}(H)=\operatorname{Alt}(k)$. The assumption that $k>4$ implies that $\operatorname{Alt}(k)$ is simple; therefore, either: (i) $\pi_{1}\left(\operatorname{ker} \pi_{2} \cap H\right)=$ $\langle 1\rangle$, or else (ii) $\pi_{1}\left(\operatorname{ker} \pi_{2} \cap H\right)=\operatorname{Alt}(k)$. But ker $\pi_{2} \cap H \leqslant \operatorname{Alt}(k) \times\langle 1\rangle$. In particular, ker $\pi_{2} \cap H=\pi_{1}\left(\operatorname{ker} \pi_{2} \cap H\right) \times\langle 1\rangle$. Thus, in case (i) we have ker $\pi_{2} \cap H=\langle 1\rangle$, and

$$
|\operatorname{Alt}(m)| \geqslant\left|\pi_{2}(H)\right|=|H| \geqslant \frac{|H|}{\left|\operatorname{ker} \pi_{1} \cap H\right|}=\left|\pi_{1}(H)\right|=|\operatorname{Alt}(k)|,
$$

a contradiction of the assumptions of the lemma.

In case (ii), we have $\operatorname{Alt}(k) \times\langle 1\rangle=\operatorname{ker} \pi_{2} \cap H \unlhd H$ and $|H|=\left|\operatorname{ker} \pi_{2} \cap H\right| \cdot\left|\pi_{2}(H)\right|=$ $|\operatorname{Alt}(k)| \cdot|B|$. Now the claim follows from the trivial inclusion $H \subseteq \operatorname{Alt}(k) \times B$.

As we agreed before, $\operatorname{Sym}(n)$ is identified with the group of permutation matrices. If $\sigma \in \operatorname{Sym}(n)$, the corresponding permutation matrix $\sum_{i=1}^{n} e_{i, i \sigma}$ is denoted by $g_{\sigma}$. We write $I_{k}$ for the $k \times k$ identity matrix. Let $r \in R$. Recall that by $R_{r}$ we denote the subring of $R$ (maybe without unity) generated by $r$ : that is, the set of all sums $c_{1} r+c_{2} r^{2}+\ldots+c_{l} r$, where $l, c_{1}, \ldots, c_{l}$ are integers. If $R=R_{r}$, we say that $r$ generates $R$.

Lemma 3.2. For $n \geqslant 3$, we have $\mathrm{SL}_{n}\left(R_{1}\right) \leqslant \mathrm{E}_{n}(R)$. In particular, $\mathrm{E}_{n}(R)$ contains $\operatorname{Alt}(n)$ and all diagonal matrices with entries \pm 1 and determinant 1 .

Proof. The ring $R_{1}$ is isomorphic to either $\mathbb{Z}$ or $\mathbb{Z} / m \mathbb{Z}$. Therefore, $\operatorname{SL}_{n}\left(R_{1}\right)=\mathrm{E}_{n}\left(R_{1}\right) \leqslant$ $\mathrm{E}_{n}(R)$, provided that $n \geqslant 3$ (see $[4,1.2 .11$ and 4.3.9]). 
Lemma 3.3. (i) Let $Q$ be the block diagonal matrix

$$
Q=\operatorname{diag}\left(P, I_{n-h-2}\right),
$$

where

$$
P=\left(\begin{array}{ccccc}
-1 & 0 & r_{1} & \ldots & r_{h} \\
0 & -1 & s_{1} & \ldots & s_{h} \\
0 & & & I_{h} &
\end{array}\right)
$$

and $r_{i}, s_{i} \in R$, for $i=1, \ldots, h$. Let $E$ be the group generated by $Q$ and $\operatorname{Alt}(n)$. Then we have $E \leqslant \mathrm{E}_{n}(R)$.

(ii) Let $n \geqslant h+5$. Suppose that for some $j_{0}, 1 \leqslant j_{0} \leqslant h$, the element $r_{j_{0}}$ generates $R$ and $s_{j_{0}}=0$. Then

$$
I_{n}+r e_{i j}-r e_{i k} \in E
$$

for any $r \in R$ and any pairwise distinct $i, j, k$, where $1 \leqslant i, j, k \leqslant n$.

(iii) Let $n \geqslant h+5$ and $j_{0}$ be as above. Set

$$
\rho=r_{j_{0}}\left(2-r_{1}-\ldots-r_{h}\right)
$$

and assume that $1 \in R_{\rho}$. Then $E=\mathrm{E}_{n}(R)$.

Proof. (i) By Lemma 3.2, $\operatorname{diag}\left(-I_{2}, I_{n-2}\right) \in \mathrm{E}_{n}(R)$ and $\operatorname{Alt}(n) \leqslant \mathrm{E}_{n}(R)$.

Now, the identity

$$
Q=\prod_{i=1}^{h}\left(I_{n}+r_{i} e_{1, i+2}\right) \prod_{i=1}^{h}\left(I_{n}+s_{i} e_{2, i+2}\right) \operatorname{diag}\left(-I_{2}, I_{n-2}\right)
$$

implies that $Q \in \mathrm{E}_{n}(R)$. Thus, $E \leqslant \mathrm{E}_{n}(R)$.

(ii) Without loss of generality, we may assume that $j_{0}=1$. (Otherwise, replace $Q$ by its conjugate $g_{\sigma_{1}}^{-1} Q g_{\sigma_{1}}$, where $\sigma_{1}=\left(3, j_{0}+2\right)(h+3, h+4)$; note that $\sigma_{1} \in \operatorname{Alt}(n)$ if $j_{0}>1$. $)$ In particular, $s_{1}=0$. Let $\sigma_{2}=(3, h+3)(h+4, h+5)$. A direct calculation shows that

$$
\begin{aligned}
Q g_{\sigma_{2}}^{-1} Q g_{\sigma_{2}}= & \left(I_{n}-2 e_{11}-2 e_{22}+r_{1} e_{13}+\sum_{i=2}^{h} r_{i} e_{1, i+2}+\sum_{i=2}^{h} s_{i} e_{2, i+2}\right) \\
& \times\left(I_{n}-2 e_{11}-2 e_{22}+r_{1} e_{1, h+3}+\sum_{i=2}^{h} r_{i} e_{1, i+2}+\sum_{i=2}^{h} s_{i} e_{2, i+2}\right) \\
= & I_{n}+r_{1} e_{13}-r_{1} e_{1, h+3} .
\end{aligned}
$$

Consequently,

$$
I_{n}+r_{1} e_{13}-r_{1} e_{1, h+3} \in E .
$$

Conjugation by $g_{\sigma_{3}}$, where $\sigma_{3}=(h+3,3,2)$, gives us

$$
I_{n}+r_{1} e_{12}-r_{1} e_{13} \in E \text {. }
$$

Taking $\sigma_{4}=(1,2)(h+4, h+5)$, we have the following identity:

$$
\left[I_{n}+\sum_{i=2}^{h+3} \alpha_{i} e_{1 i}, g_{\sigma_{4}}^{-1}\left(I_{n}+\sum_{i=3}^{h+3} \beta_{i} e_{1 i}\right) g_{\sigma_{4}}\right]=I_{n}+\sum_{i=3}^{h+3} \alpha_{2} \beta_{i} e_{1 i},
$$

which is valid for any $\alpha_{2}, \ldots, \alpha_{h+3}, \beta_{3}, \ldots, \beta_{h+3}$. 
Starting from the matrices (18) and (19), and repeatedly applying identity (20) several times, we see that $I_{n}+r_{1}^{j} e_{13}-r_{1}^{j} e_{1, h+3} \in E$ for every positive integer $j$. But $r_{1}$ generates $R$, and therefore $I_{n}+r e_{13}-r e_{1, h+3} \in E$ for any $r \in R$. Since $n \geqslant 5$ and $\operatorname{Alt}(n)$ is $(n-2)$ transitive, by conjugating by a suitable $g_{\sigma}$ we find that (16) holds for any $r \in R$ and any $i, j, k$, where $i \neq j, i \neq k$ and $j \neq k$.

(iii) As in part (ii), we may assume that $j_{0}=1$. Now consider $\sigma_{5}=(1,3, h+3)$. We have

$$
\begin{aligned}
Q g_{\sigma_{5}}^{-1} Q g_{\sigma_{5}}= & \left(I_{n}-2 e_{11}-2 e_{22}+r_{1} e_{13}+\sum_{i=2}^{h} r_{i} e_{1, i+2}+\sum_{i=2}^{h} s_{i} e_{2, i+2}\right) \\
& \times\left(I_{n}-2 e_{33}-2 e_{22}+r_{1} e_{3, h+3}+\sum_{i=2}^{h} r_{i} e_{3, i+2}+\sum_{i=2}^{h} s_{i} e_{2, i+2}\right) \\
= & I_{n}-2 e_{11}-2 e_{33}-r_{1} e_{13}+\sum_{i=2}^{h}\left(r_{i}+r_{1} r_{i}\right) e_{1, i+2}+r_{1}^{2} e_{1, h+3} \\
& +\sum_{i=2}^{h} r_{i} e_{3, i+2}+r_{1} e_{3, h+3} .
\end{aligned}
$$

Therefore, $I_{n}+2 r_{1} e_{13}-\sum_{i=2}^{h} r_{1} r_{i} e_{1, i+2}-r_{1}^{2} e_{1, h+3}=\left(Q g_{\sigma_{5}}^{-1} Q g_{\sigma_{5}}\right)^{2} \in E$. Multiplying the matrix $\left(Q g_{\sigma_{5}}^{-1} Q g_{\sigma_{5}}\right)^{2}$ by a suitable product of matrices of the form (16), namely by

$$
\left(I_{n}-2 r_{1} e_{13}+2 r_{1} e_{1, h+3}\right) \prod_{i=2}^{h}\left(I_{n}+r_{1} r_{i} e_{1, i+2}-r_{1} r_{i} e_{1, h+3}\right),
$$

we see that $E$ contains

$$
I_{n}+\rho e_{1, h+3},
$$

where $\rho$ is defined by (17). Hence $E$ also contains

$$
I_{n}+\rho e_{1,2}=g_{\sigma_{6}}^{-1}\left(I_{n}+\rho e_{1, h+3}\right) g_{\sigma_{6}},
$$

where $\sigma_{6}=(2, h+3)(h+4, h+5)$. As above, starting from the matrices defined by (21) and (22), and repeatedly applying (20), we deduce that $I_{n}+\rho^{j} e_{1, h+3} \in E$ for any $j \geqslant 1$. The assumption that $1 \in R_{\rho}$ implies that $I_{n}+e_{1, h+3} \in E$. By (16), $I_{n}+r e_{12}-r e_{1, h+3} \in E$ for any $r \in R$. Using a special case of (20), we have

$$
I_{n}+r e_{1, h+3}=\left[I_{n}+r e_{12}-r e_{1, h+3}, g_{\sigma_{4}}^{-1}\left(I_{n}+e_{1, h+3}\right) g_{\sigma_{4}}\right] \in E .
$$

Conjugating by suitable permutational matrices, we find that $E$ contains $I_{n}+r e_{i j}$ for any $r \in R$ and any $i, j$, where $1 \leqslant i \neq j \leqslant n$. Thus it coincides with $\mathrm{E}_{n}(R)$.

Now we consider the situation described in Lemma 2.6, and we introduce some further notation. From now on, we assume that $\psi: T(2,3,7) \longrightarrow \operatorname{Sym}(\Sigma) \subseteq \mathrm{GL}_{n}(R)$ is a transitive permutational representation of $T(2,3,7)$, and that $\left\{a_{1}, a_{2}\right\} \subseteq \Sigma$ is a 1-handle with respect to $\psi$. Suppose that $b_{1}, b_{2} \in\left\langle\Sigma \backslash\left\{a_{1}, a_{2}\right\}\right\rangle$, where $b_{1} \neq b_{2}$, and they satisfy assumptions (i) and (ii) of Lemma 2.6. Thus we may apply Lemma 2.6 to define a new linear representation $\tilde{\psi}$. Let $\Gamma_{1}, \Gamma_{2} \subseteq \Sigma$ be the supports of $b_{1}$ and $b_{2}$ respectively; that is, $\Gamma_{i}$ is the smallest subset of the basis $\Sigma$ such that $b_{i} \in\left\langle\Gamma_{i}\right\rangle$. Since $\psi(X)$ fixes both $b_{1}$ and $b_{2}$ and acts as a permutation on $\Sigma$, we have $\Gamma_{i} \psi(X)=\Gamma_{i}, i=1,2$. Finally, we define $\Gamma$ as follows:

$$
\Gamma=\left\{v \in \Gamma_{1} \cup \Gamma_{2}: v \psi(X) \neq v\right\}
$$


LEMMA 3.4. Under the assumptions and notations of the preceding paragraph, suppose further that for some $\Delta_{0} \subseteq \Sigma$, the following conditions are satisfied:

(i) $\left|\Delta_{0}\right| \geqslant 3$;

(ii) $\Delta_{0}$ contains at least two points from an orbit of $\psi(Y)$ and $\Delta_{0} \backslash \Gamma$ contains an orbit of $\psi(X)$ of length 2;

(iii) $\operatorname{Alt}\left(\Delta_{0}\right)$ is a subgroup of $\langle\tilde{\psi}(X), \tilde{\psi}(Y)\rangle$.

Let $\Delta$ be a maximal subset of $\Sigma$ with respect to the following properties:

$$
\Delta_{0} \subseteq \Delta \quad \text { and } \quad \operatorname{Alt}(\Delta) \leqslant\langle\tilde{\psi}(X), \tilde{\psi}(Y)\rangle
$$

Then $\Delta \psi(Y)=\Delta$ and $(\Delta \backslash \Gamma) \psi(X) \subseteq \Delta$.

Proof. Assumption (ii) yields $\Delta \cap \Delta \psi(Y) \neq \emptyset$. Recall also that $\psi(Y)=\tilde{\psi}(Y)$. By condition (i), $|\Delta| \geqslant\left|\Delta_{0}\right| \geqslant 3$ and $|\Delta \psi(Y)|=|\Delta| \geqslant 3$. This, together with (24), implies that

$$
\begin{aligned}
\operatorname{Alt}(\Delta \cup \Delta \psi(Y)) & =\langle\operatorname{Alt}(\Delta), \operatorname{Alt}(\Delta \psi(Y))\rangle \\
& =\left\langle\operatorname{Alt}(\Delta), \tilde{\psi}\left(Y^{-1}\right) \operatorname{Alt}(\Delta) \tilde{\psi}(Y)\right\rangle \leqslant\langle\tilde{\psi}(X), \tilde{\psi}(Y)\rangle .
\end{aligned}
$$

By the maximality of $\Delta$, we have $\Delta=\Delta \psi(Y)$.

Now let $\left\{w_{1}, w_{2}\right\} \subseteq \Delta_{0} \backslash \Gamma$ be an orbit of $\psi(X)$ of length two, which exists in accordance with condition (ii). Take $v \in \Delta \backslash \Gamma$. Clearly, if $v=w_{1}$ or $v=w_{2}$, then $v \psi(X) \in \Delta$. Thus we may assume that $v \neq w_{1}, w_{2}$. Hence $\left(v, w_{1}, w_{2}\right) \in \operatorname{Alt}(\Delta)$.

Recall that $a_{1}$ and $a_{2}$ are fixed points of $\psi(X)$, while $w_{1}$ and $w_{2}$ are not. We may also assume that $\psi(X)$ does not fix $v$; otherwise $v \psi(X) \in \Delta$ by trivial reasoning. By the choice of $w_{1}, w_{2}$ and $v$, we have $v, w_{1}, w_{2} \notin \Gamma$. By the definition of $\Gamma$,

$$
\Gamma_{1} \cup \Gamma_{2}=\Gamma \cup\left\{v \in \Gamma_{1} \cup \Gamma_{2}: v \psi(X)=v\right\} .
$$

Therefore, the above observations imply that $v, w_{1}, w_{2} \notin \Gamma_{1} \cup \Gamma_{2} \cup\left\{a_{1}, a_{2}\right\}$. In particular, none of $v, w_{1}$ and $w_{2}$ lies in the support of $a_{1} \tilde{\psi}(X)=a_{1} \tilde{\psi}\left(X^{-1}\right)$ or $a_{2} \tilde{\psi}(X)=a_{2} \tilde{\psi}\left(X^{-1}\right)$ (the corresponding supports are $\Gamma_{1} \cup\left\{a_{1}\right\}$ and $\Gamma_{2} \cup\left\{a_{2}\right\}$, respectively). Therefore,

$$
\tilde{\psi}\left(X^{-1}\right)\left(v, w_{1}, w_{2}\right) \tilde{\psi}(X)=\psi\left(X^{-1}\right)\left(v, w_{1}, w_{2}\right) \psi(X)=\left(v \psi(X), w_{2}, w_{1}\right) .
$$

Note that $w_{1}$ and $w_{2}$ lie in the support of $\left(v \psi(X), w_{2}, w_{1}\right)$ and in $\Delta$. Since $|\Delta| \geqslant\left|\Delta_{0}\right| \geqslant 3$, we find, using (24), that

$$
\begin{aligned}
\operatorname{Alt}(\Delta \cup\{v \psi(X)\}) & =\left\langle\operatorname{Alt}(\Delta),\left(v \psi(X), w_{2}, w_{1}\right)\right\rangle \\
& \leqslant\left\langle\operatorname{Alt}(\Delta), \tilde{\psi}\left(X^{-1}\right) \operatorname{Alt}(\Delta) \tilde{\psi}(X)\right\rangle \leqslant\langle\tilde{\psi}(X), \tilde{\psi}(Y)\rangle .
\end{aligned}
$$

By the maximality of $\Delta$, we have $v \psi(X) \in \Delta$.

COROLLARY 3.5. Under the assumptions of Lemma 3.4, suppose further that $\psi$ is a transitive permutational representation, and that $\left|\Gamma_{1}\right|=\left|\Gamma_{2}\right|=1$. Then $\langle\tilde{\psi}(X), \tilde{\psi}(Y)\rangle$ contains $\operatorname{Alt}(\Sigma)$.

Proof. If $\left|\Gamma_{1}\right|=\left|\Gamma_{2}\right|=1$, then it follows from (23) that $\Gamma$ is empty. Let $\Delta$ be a maximal set with respect to the property described in (24). By Lemma 3.4, $\Delta \psi(Y)=\Delta$ and $\Delta \psi(X)=$ $\Delta$. By the transitivity of $\langle\psi(X), \psi(Y)\rangle$ on $\Sigma$, we have $\Delta=\Sigma$.

The above corollary has already appeared as a part of the proof of [7, Theorem A], although it is not stated explicitly there. 


\section{Proof of the main theorem}

Proof of Theorem 1.3. We start from twenty basic permutational representations, which are labelled $A, \ldots, T$. The corresponding generators $X_{A}, Y_{A}, \ldots, X_{T}, Y_{T}$, together with their degrees and available handles, are listed in Appendix A. The first fourteen representations $(A, \ldots, N)$ are extracted from Conder's list of coset diagrams [1]. The remaining ones are actually mentioned in [9], although the generators are not written there explicitly.

Here we present the general scheme of the proof, while all the necessary computational details can be read from Appendix B. The calculations were performed using the MAGMA package. Related libraries are provided in Appendix C.

Let $n$ be one of the numbers listed in the statement of Theorem 1.3, and let

$$
\Sigma=\left\{v_{1}, \ldots, v_{n}\right\} .
$$

To simplify the descriptions of permutation generation below, we identify $\Sigma$ with $\{1, \ldots, n\}$ in a natural way. Connecting some of the basic diagrams as described in Lemma 2.2, we build a permutational representation $\psi: T(2,3,7) \longrightarrow \operatorname{Sym}(\Sigma)$ of degree $n$ with at least two 1-handles, say $\left\{a_{1}, a_{2}\right\}$ and $\left\{c_{1}, c_{2}\right\}$. We use the following notation. For example, $G(1) E(2) D$ means that the representations $G$ and $E$ are joined via 1-handles, and the resulting representation is connected to $D$ via 2-handles. For a basic representation $\mathcal{D}$ of degree $d$,

$$
X_{\mathscr{D}}^{[k, k+d-1]} \quad \text { and } \quad Y_{\mathscr{D}}^{[k, k+d-1]}
$$

denote the results of the natural embeddings of $X_{\mathscr{D}}$ and $Y_{\mathscr{D}}$, respectively, into $\operatorname{Sym}\left(\left\{v_{k}, \ldots, v_{k+d-1}\right\}\right)$. Set also $X_{n}=\psi(X)$ and $Y_{n}=\psi(Y)$. Thus, in the above example the corresponding Hurwitz generators can be written as

$$
\begin{aligned}
X_{92} & =X_{G}^{[1,42]}(25,68)(26,69) X_{E}^{[43,70]}(52,71)(55,74) X_{D}^{[71,92]}, \\
Y_{92} & =Y_{G}^{[1,42]} Y_{E}^{[43,70]} Y_{D}^{[71,92]},
\end{aligned}
$$

while the free handles are $\left(a_{1}, a_{2}\right)=(1,2)_{1}$ and $\left(c_{1}, c_{2}\right)=(13,14)_{1}$.

Let $t$ be an element of $R$ that satisfies the hypothesis of Theorem 1.3. In particular, one can take $t=1$ if $R=\mathbb{Z}$, and any generator $t \neq 2$ of $\mathbb{F}_{q}$ if $R=\mathbb{F}_{q}$. Letting $b_{1}=t c_{1}$ and $b_{2}=t c_{2}$, we apply the transformation described in Lemma 2.6, and we obtain new Hurwitz generators $\tilde{\psi}(X)=U \psi(X)$ and $\tilde{\psi}(Y)=\psi(Y)=Y_{n}$. Set

$$
G=\langle\tilde{\psi}(X), \tilde{\psi}(Y)\rangle \text {. }
$$

We claim that in each case under consideration, $G=\mathrm{E}_{n}(R)$. Clearly, $\psi(Y)$ and $\psi(X Y)$ are even permutations, as their orders are odd. So $\psi(X)$ is also even. Notice that $U$ is a product of two elementary matrices and a diagonal matrix with entries \pm 1 and determinant 1 . Thus, by Lemma $3.2, \mathrm{E}_{n}(R)$ contains $\psi(X), \psi(Y)$ and $U$. Hence, $G \leqslant \mathrm{E}_{n}(R)$.

Our next aim is to prove the converse inclusion in each case. Reading the data from Appendix B, we see that in each case the assumptions of Lemmas 2.9 and 2.10 are satisfied. In fact, conditions (7) and (8) hold automatically, since $\psi$ is a permutational representation acting on $\Sigma$ such that $\psi(Y)$ permutes $a_{1}, a_{2}$ and $a_{3}$, and $\psi(X)$ permutes $a_{3}$ and $a_{4}$ and fixes $a_{1}$ and $a_{2}$. Let $C=[\psi(X), \psi(Y)]$ and $\tilde{C}=[\tilde{\psi}(X), \tilde{\psi}(Y)]$ be the commutators introduced in Lemma 2.9. Using Lemmas 2.10 and 2.11, we find $d$ such that $\tilde{C}^{d}=C^{d}$ and the support of $C^{d}$ is large enough. Set $S_{1}=C^{d}$ and $S_{2}=Y_{n}^{-1} C^{d} Y_{n}$, and let $K$ be a subgroup of $\operatorname{Sym}(n)=\operatorname{Sym}(\Sigma)$ generated by $S_{1}$ and $S_{2}$. Let $\Delta_{0}$ be the largest orbit of $K$, and let $\Delta_{1}$ be the union of all other non-trivial orbits (that is, orbits containing at least two points). We 
denote by $\bar{S}_{i}, i=1,2$, the restriction of $S_{i}$ to $\Delta_{0}$. It turns out that in all the cases below, both $\bar{S}_{1}$ and $\bar{S}_{2}$ are even permutations. If $\Delta_{1}$ is not empty, then the restriction of $S_{i}$ to $\Delta_{1}$ is given by $S_{i} \bar{S}_{i}^{-1}$. Since $S_{1}$ and $S_{2}$, as powers of commutators, are even, both $S_{1} \bar{S}_{1}^{-1}$ and $S_{2} \bar{S}_{2}^{-1}$ are also even. Therefore, $K \leqslant \operatorname{Alt}\left(\Delta_{0}\right) \times \operatorname{Alt}\left(\Delta_{1}\right)$. Set $\bar{K}=\left\langle\bar{S}_{1}, \bar{S}_{2}\right\rangle$. In each of the cases under consideration, we will find an element $W$ of $\bar{K}$, some power of which is a cycle of a prime length $\ell$ with $\left|\Delta_{0}\right| / 2<\ell<\left|\Delta_{0}\right|-3$. The first inequality guarantees that $\bar{K}$ is primitive on $\Delta_{0}$, and hence the second inequality, combined with a well-known theorem of Jordan [5] (see [11, p. 39]) implies that $\bar{K}=\operatorname{Alt}\left(\Delta_{0}\right)$. It turns out that in all the cases under consideration, $\left|\Delta_{1}\right|<\left|\Delta_{0}\right|$. If $\Delta_{1}=\emptyset$, then $\operatorname{Alt}\left(\Delta_{0}\right)=\bar{K}=K$. Otherwise, $\operatorname{Alt}\left(\Delta_{0}\right) \leqslant K$, by Lemma 3.1. In any case, we conclude that $\operatorname{Alt}\left(\Delta_{0}\right) \leqslant G$.

Next, we will check (see the relevant data in Appendix B) that $\Delta_{0}$ satisfies the assumptions of Lemma 3.4 and Corollary 3.5. Thus, Corollary 3.5 implies that

$$
\operatorname{Alt}(\Sigma) \leqslant G
$$

Therefore, $G$ contains $\psi(X)$ and $U=\tilde{\psi}(X) \psi(X)$. Conjugating $U$ by a suitable permutational matrix, we find that $G$ contains a matrix $Q$ of the form (15), where the block $P$ is given by

$$
\left(\begin{array}{rrrr}
-1 & 0 & t & 0 \\
0 & -1 & 0 & t \\
0 & 0 & 1 & 0 \\
0 & 0 & 0 & 1
\end{array}\right)
$$

Using (25) and Lemma 3.3, we obtain the desired inclusion: $\mathrm{E}_{n}(R) \leqslant G$. Hence $\mathrm{E}_{n}(R)=G$.

To complete the proof, we collect the relevant data from Appendix B. For each $n$ listed in the statement of Theorem 1.3, the following information is presented:

- the description of the representation $\psi$;

- the generators $X_{n}$ and $Y_{n}$;

- 1-handles $\left(a_{1}, a_{2}\right)$ and $\left(c_{1}, c_{2}\right)$, as well as $a_{3}=a_{2} \psi(Y), a_{4}=a_{3} \psi(X), c_{3}=c_{2} \psi(Y)$ and $c_{5}=c_{1} \psi\left(Y^{-1} X Y\right)=c_{3} \psi(X Y)$;

- the cycle structure of the commutator $C=[\psi(X), \psi(Y)]$ (to avoid any confusion with the name of one of the basic representations, we write $[\psi(X), \psi(Y)]$ for the commutator in Appendix B); in addition, $a_{1}, a_{4}, c_{3}$ and $c_{5}$ are printed in bold;

- the values of $r$ and $s$ used in Lemmas 2.10 and 2.11 (if $r=s$, then $k$ such that $a_{1} C^{k}=a_{4}, c_{5} C^{k}=c_{3}$ is also indicated);

- the degree $d$ such that $C^{d}=\tilde{C}^{d}$;

- the set $\Delta_{0}$ and the lengths of other non-trivial orbits of $K$ (if any);

- an orbit $\left\{w_{1}, w_{2}\right\}$ of $\psi(X)$, and two points $w_{3}, w_{4}$ from an orbit of $\psi(Y)$ lying in $\Delta_{0}$;

- the cycle structure of $\bar{S}_{1}$ (which coincides with the cycle structure of $\bar{S}_{2}$ );

- an element $W$ of $\bar{K}$, represented as a word in $\bar{S}_{1}, \bar{S}_{2}$, together with its cycle structure (a cycle of large prime length is printed in bold).

To represent the cycle structure of a permutation, we use the following notation:

$$
\sigma=\left(i_{1}, i_{2}, \ldots\right)\left(j_{1}, j_{2} \ldots\right) \ell_{1}^{\alpha_{1}} \ldots \ell_{s}^{\alpha_{s}}
$$

means that $\sigma$ has cycles $\left(i_{1}, i_{2}, \ldots\right),\left(j_{1}, j_{2} \ldots\right)$ and also $\alpha_{h}$ cycles of length $\ell_{h}, h=1, \ldots, s$. An analysis of the data in Appendix B finishes the proof. 
To complete this section, we discuss the range of applicability of the method described in the proof of Theorem 1.3. For this purpose, we deduce from a result of Scott [8], an inequality similar to the well-known genus formula.

Let $\psi: T(2,3,7) \longrightarrow \operatorname{Sym}(\Sigma) \subseteq \mathrm{GL}_{n}(\mathbb{C})$ be a transitive permutational representation. By $d_{X}, d_{Y}, d_{X Y}$ we denote the dimension of the subspace of $\mathbb{C}^{n}$ fixed by $\psi(X)$, $\psi(Y)$, and $\psi(X Y)$, respectively. In addition, let $d$ be the dimension of the subspace fixed by $\psi(T(2,3,7))$. Since $\psi$ is a transitive representation, we have $d=1$. Since $\psi$ is a permutational representation, it coincides with its dual. Thus, Scott's formula [8] becomes

$$
d_{X}+d_{Y}+d_{X Y} \leqslant n+2 d=n+2
$$

On the other hand,

$$
d_{Y} \geqslant n-2\left\lfloor\frac{n}{3}\right\rfloor, \quad d_{X Y} \geqslant n-6\left\lfloor\frac{n}{7}\right\rfloor .
$$

The above proof of Theorem 1.3 requires at least two 1-handles, so $\psi(X)$ fixes at least four points in $\Sigma$. On the other hand, $\psi(X)=\psi(X Y) \psi\left(Y^{-1}\right)$ must be an even permutation. Therefore,

$$
d_{X} \geqslant n-2\left\lfloor\frac{n-4}{4}\right\rfloor .
$$

By (26), we have

$$
2 n-2 \leqslant 2\left\lfloor\frac{n-4}{4}\right\rfloor+2\left\lfloor\frac{n}{3}\right\rfloor+6\left\lfloor\frac{n}{7}\right\rfloor .
$$

The only numbers $n \leqslant 286$ that satisfy (27) but whose status still remains open, are $21,28,36,42,56,72$, and 144. For example, the representation $S(2) M$ of degree 144 has two 1-handles, but the commutator $C$ does not satisfy assumption (ii) of Lemma 2.10. This case deserves special treatment.

\section{Appendix A. Basic representations and their generators}

1. Representation $A$ of degree 14; one handle: $(1,2)_{1}$.

$X_{A}=(3,4)(5,7)(6,10)(8,12)(9,14)(11,13)(1)(2)$,

$Y_{A}=\prod_{i=0}^{3}(3 i+1,3 i+2,3 i+3)(13)(14)$.

2. Representation $B$ of degree 15 ; one handle: $(4,8)_{3}$ or $(1,4)_{2}$ or $(8,1)_{2}$.

$X_{B}=(2,6)(3,9)(5,11)(7,12)(10,13)(14,15)(1)(4)(8)$,

$Y_{B}=\prod_{i=0}^{4}(3 i+1,3 i+2,3 i+3)$.

3. Representation $C$ of degree 21 ; two handles: $(1,2)_{1}$ and $(8,14)_{3}$.

$X_{C}=(3,4)(5,16)(6,20)(7,18)(9,10)(11,13)(15,21)(17,19)(1)(2)(8)(12)(14)$,

$Y_{C}=\prod_{i=0}^{6}(3 i+1,3 i+2,3 i+3)$.

4. Representation $D$ of degree 22; one handle: $(1,4)_{2}$.

$X_{D}=(2,6)(3,10)(5,7)(8,13)(9,19)(11,20)(12,14)(15,16)(17,18)(21,22)(1)(4)$,

$Y_{D}=\prod_{i=0}^{6}(3 i+1,3 i+2,3 i+3)(22)$. 
5. Representation $E$ of degree 28 ; two handles: $(26,27)_{1}$ and $(10,13)_{2}$.

$X_{E}=(1,28)(2,7)(3,4)(5,12)(6,19)(8,23)(9,14)(11,15)(16,20)(17,22)(18,25)$ $(21,24)(10)(13)(26)(27)$,

$Y_{E}=\prod_{i=0}^{8}(3 i+1,3 i+2,3 i+3)(28)$.

6. Representation $F$ of degree 30 ; one handle: $(1,4)_{2}$.

$X_{F}=(2,6)(3,10)(5,7)(8,13)(9,27)(11,20)(12,14)(15,16)(17,21)(18,26)(19,22)$ $(23,24)(25,28)(29,30)(1)(4)$,

$Y_{F}=\prod_{i=0}^{9}(3 i+1,3 i+2,3 i+3)$.

7. Representation $G$ of degree 42 ; three handles: $(1,2)_{1},(13,14)_{1}$ and $(25,26)_{1}$.

$X_{G}=(3,4)(5,11)(6,7)(8,40)(9,12)(10,37)(15,16)(17,23)(18,19)(20,41)(21,24)$ $(22,39)(27,28)(29,34)(30,31)(32,42)(33,35)(36,38)(1)(2)(13)(14)(25)(26)$,

$Y_{G}=\prod_{i=0}^{13}(3 i+1,3 i+2,3 i+3)$.

8. Representation $H$ of degree 42 ; two handles: $(1,2)_{1}$, and $(24,27)_{3}$.

$X_{H}=(3,4)(5,10)(6,7)(8,30)(9,11)(12,14)(13,16)(15,34)(17,25)(18,21)(19,23)$ $(20,28)(22,32)(26,33)(29,41)(31,39)(35,37)(38,40)(1)(2)(24)(27)(36)(42)$,

$Y_{H}=\prod_{i=0}^{13}(3 i+1,3 i+2,3 i+3)$.

9. Representation $I$ of degree 57 ; two handles: $(4,7)_{2}$, and $(36,39)_{2}$.

$X_{I}=(2,12)(3,15)(5,9)(6,13)(8,11)(10,18)(14,24)(16,55)(17,19)(20,27)(21,22)$ $(23,56)(25,30)(26,33)(28,40)(29,37)(31,35)(32,50)(34,38)(41,52)(42,43)$ $(44,48)(45,51)(46,47)(49,54)(53,57)(1)(4)(7)(36)(39)$,

$Y_{I}=\prod_{i=0}^{18}(3 i+1,3 i+2,3 i+3)$.

10. Representation $J$ of degree 72 ; two handles: $(1,2)_{1}$, and $(62,63)_{1}$.

$X_{J}=(3,4)(5,12)(6,8)(7,10)(9,15)(11,30)(13,36)(14,16)(17,31)(18,21)(19,24)$ $(20,27)(22,23)(25,33)(26,28)(29,39)(32,42)(34,38)(35,57)(37,60)(40,45)$ $(41,54)(43,56)(44,49)(46,51)(47,48)(50,52)(53,58)(55,64)(59,71)(61,69)$ $(65,67)(66,72)(68,70)(1)(2)(62)(63)$,

$Y_{J}=\prod_{i=0}^{23}(3 i+1,3 i+2,3 i+3)$.

11. Representation $K$ of degree 72 ; one handle: $(1,2)_{1}$.

$X_{K}=(3,4)(5,10)(6,7)(8,24)(9,11)(12,15)(13,33)(14,16)(17,27)(18,21)(19,30)$ $(20,22)(23,56)(25,39)(26,48)(28,69)(29,63)(31,52)(32,34)(35,37)(38,40)$ $(41,46)(42,43)(44,45)(47,51)(49,54)(50,67)(53,55)(57,59)(58,62)(61,65)$ $(64,68)(66,70)(71,72)(1)(2)(36)(60)$,

$Y_{K}=\prod_{i=0}^{23}(3 i+1,3 i+2,3 i+3)$.

12. Representation $L$ of degree 102 ; one handle: $(1,4)_{2}$.

$X_{L}=(2,6)(3,10)(5,7)(8,13)(9,20)(11,24)(12,14)(15,16)(17,63)(18,35)(19,27)$

$(21,30)(22,26)(23,48)(25,81)(28,32)(29,64)(31,41)(33,34)(36,38)(37,40)$

$(39,82)(42,43)(44,45)(46,95)(47,49)(50,52)(51,61)(53,58)(54,55)(56,57)$ $(59,86)(60,62)(65,75)(66,69)(67,78)(68,72)(70,74)(71,79)(73,84)(76,77)$ $(80,91)(83,85)(87,88)(89,94)(90,92)(93,99)(96,98)(97,100)(101,102)(1)(4)$, $Y_{L}=\prod_{i=0}^{33}(3 i+1,3 i+2,3 i+3)$. 
13. Representation $M$ of degree 108; two handles: $(1,2)_{1}$ and $(82,85)_{2}$.

$X_{M}=(3,4)(5,14)(6,7)(8,10)(9,15)(11,24)(12,31)(13,17)(16,21)(18,25)(19,64)$ $(20,22)(23,47)(26,28)(27,53)(29,33)(30,76)(32,34)(35,37)(36,49)(38,43)$ $(39,40)(41,42)(44,46)(45,50)(48,98)(51,89)(52,56)(54,68)(55,61)(57,58)$ $(59,60)(62,67)(63,65)(66,94)(69,70)(71,73)(72,91)(74,78)(75,84)(77,79)$ $(80,88)(81,86)(83,87)(90,92)(93,100)(95,102)(96,103)(97,101)(99,105)$ $(104,106)(107,108)(1)(2)(82)(85)$,

$Y_{M}=\prod_{i=0}^{35}(3 i+1,3 i+2,3 i+3)$.

14. Representation $N$ of degree 108 ; two handles: $(1,2)_{1}$ and $(33,35)_{3}$.

$X_{N}=(3,4)(5,10)(6,7)(8,14)(9,11)(12,25)(13,17)(15,59)(16,20)(18,44)(19,23)$ $(21,28)(22,26)(24,67)(27,82)(29,31)(30,34)(32,37)(36,62)(38,40)(39,63)$ $(41,43)(42,98)(45,46)(47,49)(48,88)(50,55)(51,52)(53,54)(56,58)(57,89)$ $(60,84)(61,65)(64,68)(66,94)(69,71)(70,74)(72,86)(73,80)(75,76)(77,78)$ $(79,83)(81,85)(87,93)(90,92)(91,102)(95,103)(96,100)(97,104)(99,101)$ $(105,106)(107,108)(1)(2)(33)(35)$,

$Y_{N}=\prod_{i=0}^{35}(3 i+1,3 i+2,3 i+3)$.

15. Representation $O$ of degree 7 ; one handle: $(5,6)_{1}$ or $(1,5)_{2}$ or $(1,6)_{3}$.

$X_{O}=(2,4)(3,7)(1)(5)(6)$,

$Y_{O}=\prod_{i=0}^{1}(3 i+1,3 i+2,3 i+3)(7)$.

16. Representation $P$ of degree 15 ; one handle: $(1,2)_{1}$. $X_{P}=(3,4)(5,10)(6,7)(9,11)(12,13)(14,15)(1)(2)(8)$,

$Y_{P}=\prod_{i=0}^{4}(3 i+1,3 i+2,3 i+3)$.

17. Representation $Q$ of degree 21 ; two handles: $(1,2)_{1}$ and $(15,18)_{2}$.

$X_{Q}=(3,4)(5,9)(6,11)(7,10)(8,21)(12,16)(13,17)(14,19)(1)(2)(15)(18)(20)$, $Y_{Q}=\prod_{i=0}^{6}(3 i+1,3 i+2,3 i+3)$.

18. Representation $R$ of degree 22 ; one handle: $(1,2)_{1}$. $X_{R}=(3,4)(5,12)(6,7)(8,20)(9,10)(11,17)(13,18)(14,15)(16,19)(21,22)(1)(2)$, $Y_{R}=\prod_{i=0}^{6}(3 i+1,3 i+2,3 i+3)(22)$.

19. Representation $S$ of degree 36 ; two handles: $(1,2)_{1}$ and $(14,17)_{2}$.

$X_{S}=(3,4)(5,35)(6,7)(8,10)(9,36)(11,22)(12,13)(15,16)(18,19)(20,24)(21,33)$ $(23,25)(26,31)(27,28)(29,30)(32,34)(1)(2)(14)(17)$,

$Y_{S}=\prod_{i=0}^{11}(3 i+1,3 i+2,3 i+3)$.

20. Representation $T$ of degree 66 ; one handle: $(1,2) 1$.

$X_{T}=(3,4)(5,12)(6,7)(8,33)(9,10)(11,13)(14,42)(15,16)(17,19)(18,30)(20,57)$ $(21,22)(23,28)(24,25)(26,27)(29,31)(32,34)(35,60)(36,37)(38,48)(39,40)$ $(41,43)(44,46)(45,52)(47,49)(50,51)(53,61)(54,55)(56,58)(59,62)(63,64)$ $(65,66)(1)(2)$,

$Y_{T}=\prod_{i=0}^{21}(3 i+1,3 i+2,3 i+3)$. 


\section{Appendix B. Data used in the proof of Theorem 1.3}

$n=286$. Representation $J(1) G(1) G(1) E(2) L$.

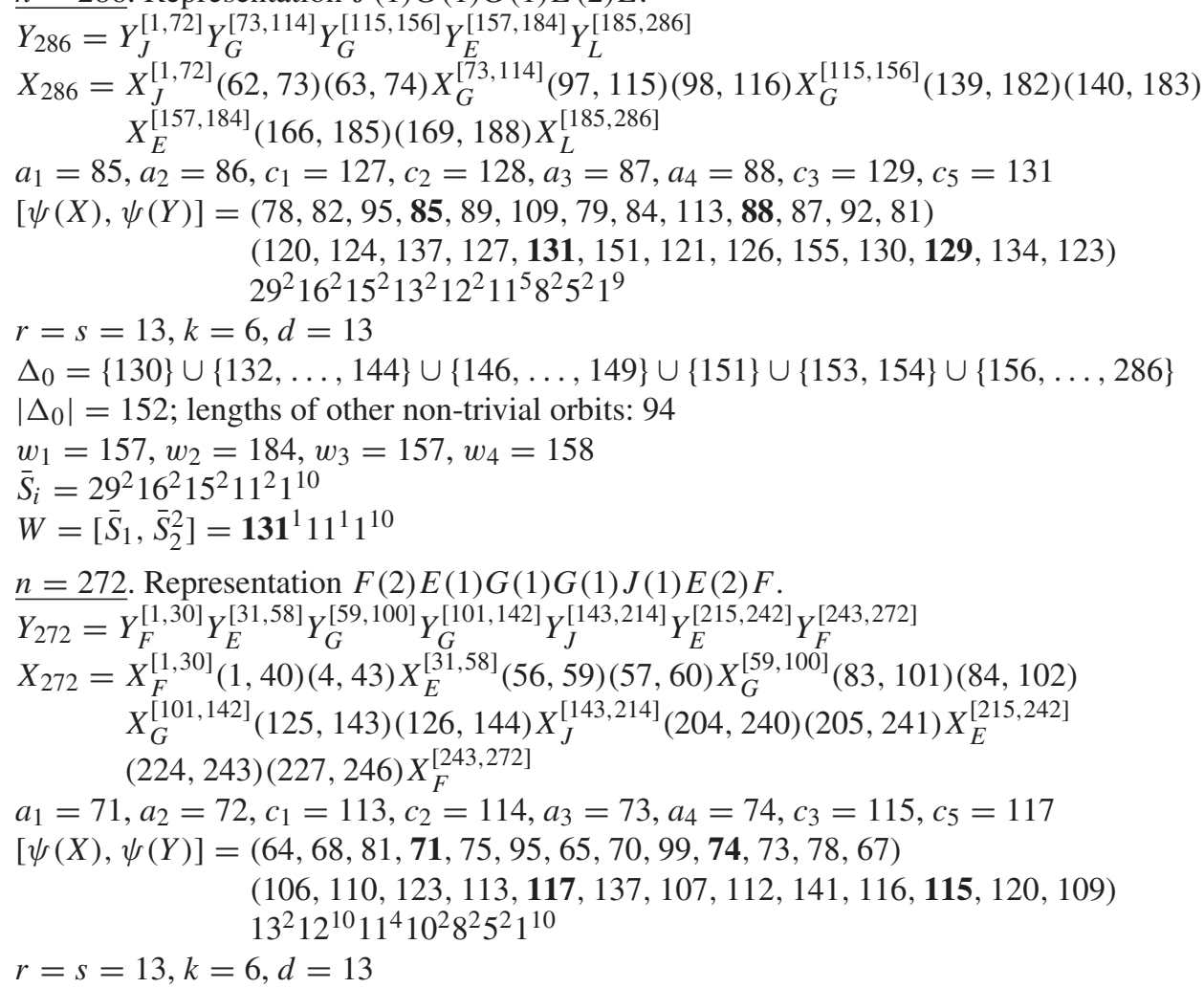

$\Delta_{0}=\{116\} \cup\{118, \ldots, 130\} \cup\{132, \ldots, 135\} \cup\{137\} \cup\{139,140\} \cup\{142, \ldots, 272\}$

$\left|\Delta_{0}\right|=152$; lengths of other non-trivial orbits: 80

$w_{1}=157, w_{2}=151, w_{3}=157, w_{4}=155$

$\bar{S}_{i}=12^{6} 11^{2} 10^{2} 8^{2} 5^{2} 1^{12}$

$W=\left[\bar{S}_{1}^{2}, \bar{S}_{2}^{6}\right]=\mathbf{1 0 1}^{1} 21^{2} 3^{1} 1^{6}$.

$\underline{n=265}$. Representation $J(1) M(2) I(2) E$.

$Y_{265}=Y_{J}^{[1,72]} Y_{M}^{[73,180]} Y_{I}^{[181,237]} Y_{E}^{[238,265]}$

$X_{265}=X_{J}^{[1,72]}(62,73)(63,74) X_{M}^{[73,180]}(154,184)(157,187) X_{I}^{[181,237]}(216,247)$

$(219,250) X_{E}^{[238,265]}$

$a_{1}=1, a_{2}=2, c_{1}=263, c_{2}=264, a_{3}=3, a_{4}=4, c_{3}=262, c_{5}=253$

$[\psi(X), \psi(Y)]=(\mathbf{1}, 5,28,31,15, \mathbf{4}, 3,9,17,26,12)$

$(240,257,263, \mathbf{2 5 3}, 241,245,255, \mathbf{2 6 2}, 260)$

$55^{1} 16^{2} 13^{2} 12^{2} 11^{2} 8^{4} 7^{2} 6^{2} 5^{4} 2^{2} 1^{4}$

$s=11, r=9, d=99$

$\Delta_{0}=\{4\} \cup\{6, \ldots, 14\} \cup\{16,18,19,21,25,26,28\} \cup\{30, \ldots, 34\} \cup\{36,37,38\}$ $\cup\{40, \ldots, 45\} \cup\{50, \ldots, 252\} \cup\{254, \ldots, 261\} \cup\{265\}$

$\left|\Delta_{0}\right|=243$; no other non-trivial orbits

$w_{1}=6, w_{2}=8, w_{3}=6, w_{4}=4$

$\bar{S}_{i}=16^{2} 13^{2} 8^{4} 7^{2} 5^{15} 4^{6} 2^{8} 1^{24}$

$W=\left[\bar{S}_{1}^{2}, \bar{S}_{2}^{4}\right]=151^{1} 37^{1} 5^{1} 3^{3} 1^{41}$. 
$n=257$. Representation $F(2) E(1) G(1) G(1) E(2) I(2) F$.

$Y_{257}=Y_{F}^{[1,30]} Y_{E}^{[31,58]} Y_{G}^{[59,100]} Y_{G}^{[101,142]} Y_{E}^{[143,170]} Y_{I}^{[171,227]} Y_{F}^{[228,257]}$
$X_{257}=X_{F}^{[1,30]}(1,40)(4,43) X_{E}^{[31,58]}(56,59)(57,60) X_{G}^{[59,100]}(83,101)(84,102)$
$\quad X_{G}^{[101,142]}(125,168)(126,169) X_{E}^{[143,170]}(152,174)(155,177) X_{I}^{[171,227]}$
$\quad(206,228)(209,231) X_{F}^{[228,257]}$
$a_{1}=71, a_{2}=72, c_{1}=113, c_{2}=114, a_{3}=73, a_{4}=74, c_{3}=115, c_{5}=117$
$[\psi(X), \psi(Y)]=(64,68,81,71,75,95,65,70,99,74,73,78,67)$

$(106,110,123,113, \mathbf{1 1 7}, 137,107,112,141,116, \mathbf{1 1 5}, 120,109)$

$17^{1} 15^{2} 13^{2} 12^{4} 11^{8} 5^{2} 2^{2} 1^{8}$

$s=13, r=13, k=6, d=13$

$\Delta_{0}=\{116\} \cup\{118, \ldots, 130\} \cup\{132, \ldots, 135\} \cup\{137,139,140\} \cup\{142, \ldots, 257\}$

$\left|\Delta_{0}\right|=137$; lengths of other non-trivial orbits: 80

$w_{1}=254, w_{2}=236, w_{3}=157, w_{4}=155$

$\bar{S}_{i}=17^{1} 15^{2} 11^{6} 5^{2} 2^{2} 1^{10}$

$W=\left[\bar{S}_{1}, \bar{S}_{2}^{2}\right]=\mathbf{7 3}^{1} 15^{1} 10^{2} 9^{1} 5^{2} 1^{10}$.

$\underline{n=250}$. Representation $G(1) J(1) M(2) E$.

$Y_{250}=Y_{G}^{[1,42]} Y_{J}^{[43,114]} Y_{M}^{[115,222]} Y_{E}^{[223,250]}$

$X_{250}=X_{G}^{[1,42]}(25,43)(26,44) X_{J}^{[43,114]}(104,115)(105,116) X_{M}^{[115,222]}(196,232)$ $(199,235) X_{E}^{[223,250]}$

$a_{1}=1, a_{2}=2, c_{1}=13, c_{2}=14, a_{3}=3, a_{4}=4, c_{3}=15, c_{5}=17$

$[\psi(X), \psi(Y)]=(\mathbf{1}, 5,38,31,35,40, \mathbf{4}, 3,8,33,30,36,11)$

$(6,10,23,13, \mathbf{1 7}, 37,7,12,41,16, \mathbf{1 5}, 20,9)$

$28^{2} 14^{2} 13^{2} 12^{2} 11^{2} 9^{1} 8^{2} 7^{2} 6^{2} 5^{2} 1^{7}$

$s=13, r=13, k=6, d=13$

$\Delta_{0}=\{16\} \cup\{18, \ldots, 30\} \cup\{32, \ldots, 35\} \cup\{37,39,40\} \cup\{42, \ldots, 150\} \cup\{152,153\}$

$\cup\{157, \ldots, 168\} \cup\{170,171\} \cup\{175, \ldots, 218\} \cup\{223, \ldots, 250\}$

$\left|\Delta_{0}\right|=218$; no other non-trivial orbits

$w_{1}=211, w_{2}=215, w_{3}=211, w_{4}=212$

$\bar{S}_{i}=28^{2} 14^{2} 12^{2} 11^{2} 9^{1} 8^{2} 7^{2} 6^{2} 5^{2} 1^{27}$

$W=\left[\bar{S}_{1}^{2}, \bar{S}_{2}^{4}\right]=167^{1} 27^{1} 3^{2} 1^{18}$.

$n=244$. Representation $J(1) G(1) E(2) L$.

$Y_{244}=Y_{J}^{[1,72]} Y_{G}^{[73,114]} Y_{E}^{[115,142]} Y_{L}^{[143,244]}$

$X_{244}=X_{J}^{[1,72]}(62,73)(63,74) X_{G}^{[73,114]}(97,140)(98,141) X_{E}^{[115,142]}(124,143)(127,146)$ $X_{L}^{[143,244]}$

$a_{1}=1, a_{2}=2, c_{1}=85, c_{2}=86, a_{3}=3, a_{4}=4, c_{3}=87, c_{5}=89$

$[\psi(X), \psi(Y)]=(\mathbf{1}, 5,28,31,15, \mathbf{4}, 3,9,17,26,12)$

$(78,82,95,85, \mathbf{8 9}, 109,79,84,113,88, \mathbf{8 7}, 92,81)$

$29^{2} 16^{2} 15^{2} 12^{2} 11^{4} 8^{2} 5^{2} 1^{6}$

$s=11, r=13, d=143$

$\Delta_{0}=\{115, \ldots, 129\} \cup\{131, \ldots, 138\} \cup\{142, \ldots, 244\}$

$\left|\Delta_{0}\right|=126$; lengths of other non-trivial orbits: 76

$w_{1}=127, w_{2}=146, w_{3}=127, w_{4}=128$

$\bar{S}_{i}=29^{2} 16^{2} 15^{2} 1^{6}$

$W=\left[\bar{S}_{1}^{2}, \bar{S}_{2}^{3}\right]=\mathbf{7 9}^{1} 37^{1} 2^{2} 1^{6}$. 
$\underline{n=243}$. Representation $B(3) N(1) G(1) G(1) C(3) B$.

$\begin{aligned} Y_{243}= & Y_{B}^{[1,15]} Y_{N}^{[16,123]} Y_{G}^{[124,165]} Y_{G}^{[166,207]} Y_{C}^{[208,228]} Y_{B}^{[229,243]} \\ X_{243}= & X_{B}^{[1,15]}(4,48)(8,50) X_{N}^{[16,123]}(16,124)(17,125) X_{G}^{[124,165]}(148,166)(149,167) \\ & X_{G}^{[166,207]}(190,208)(191,209) X_{C}^{[208,228]}(215,232)(221,236) X_{B}^{[229,243]}\end{aligned}$

$a_{1}=136, a_{2}=137, c_{1}=178, c_{2}=179, a_{3}=138, a_{4}=139, c_{3}=180, c_{5}=182$

$[\psi(X), \psi(Y)]=(129,133,146, \mathbf{1 3 6}, 140,160,130,135,164, \mathbf{1 3 9}, 138,143,132)$

$(171,175,188,178, \mathbf{1 8 2}, 202,172,177,206,181, \mathbf{1 8 0}, 185,174)$

$23^{2} 17^{2} 13^{2} 12^{2} 11^{1} 10^{2} 9^{2} 6^{2} 5^{2} 4^{2} 1^{8}$

$s=13, r=13, k=6, d=13$

$\Delta_{0}=\{1, \ldots, 129\} \cup\{131,132,134,135,151,\} \cup\{153, \ldots, 159\} \cup\{161, \ldots, 164\}$

$\left|\Delta_{0}\right|=145$; lengths of other non-trivial orbits: 58

$w_{1}=158, w_{2}=156, w_{3}=157, w_{4}=158$

$\bar{S}_{i}=23^{2} 17^{2} 10^{2} 9^{2} 6^{2} 5^{1} 1^{10}$

$W=\left[\bar{S}_{1}, \bar{S}_{2}^{3}\right]=\mathbf{1 3 1}^{1} 6^{2} 1^{2}$.

$n=236$. Representation $A(1) G(1) G(1) M(2) F$.

$Y_{236}=Y_{A}^{[1,14]} Y_{G}^{[15,56]} Y_{G}^{[57,98]} Y_{M}^{[99,206]} Y_{F}^{[207,236]}$

$X_{236}=X_{A}^{[1,14]}(1,15)(2,16) X_{G}^{[15,56]}(39,57)(40,58) X_{G}^{[57,98]}(81,99)(82,100) X_{M}^{[99,206]}$

$(180,207)(183,210) X_{F}^{[207,236]}$

$a_{1}=27, a_{2}=28, c_{1}=69, c_{2}=70, a_{3}=29, a_{4}=30, c_{3}=71, c_{5}=73$

$[\psi(X), \psi(Y)]=(20,24,37, \mathbf{2 7}, 31,51,21,26,55, \mathbf{3 0}, 29,34,23)$

$(62,66,79,69,73,93,63,68,97,72,71,76,65) 32^{2} 17^{2} 13^{6} 7^{2} 6^{2} 1^{8}$

$s=13, r=13, k=6, d=13$

$\Delta_{0}=\{72\} \cup\{74, \ldots, 86\} \cup\{88, \ldots, 91\} \cup\{93,95,96\} \cup\{98, \ldots, 134\} \cup\{126,137\}$

$\cup\{141, \ldots, 152\} \cup\{154,155\} \cup\{159, \ldots, 202\} \cup\{207, \ldots, 236\}$

$\left|\Delta_{0}\right|=148$; no other non-trivial orbits

$w_{1}=160, w_{2}=165, w_{3}=159, w_{4}=160$

$\bar{S}_{i}=32^{2} 17^{2} 7^{2} 6^{2} 1^{24}$

$W=\left[\bar{S}_{1}, \bar{S}_{2}^{6}\right]=\mathbf{8 3}^{1} 41^{1} 3^{2} 1^{18}$.

$\underline{n=235}$. Representation $C(1) G(1) G(1) E(2) L$.

$Y_{235}=Y_{C}^{[1,21]} Y_{G}^{[22,63]} Y_{G}^{[64,105]} Y_{E}^{[106,133]} Y_{L}^{[134,235]}$

$X_{235}=X_{C}^{[1,21]}(1,22)(2,23) X_{G}^{[22,63]}(46,64)(47,65) X_{G}^{[64,105]}(88,131)(89,132) X_{E}^{[106,133]}$

$(115,134)(118,137) X_{L}^{[134,235]}$

$a_{1}=34, a_{2}=35, c_{1}=76, c_{2}=77, a_{3}=36, a_{4}=37, c_{3}=78, c_{5}=80$

$[\psi(X), \psi(Y)]=(27,31,44, \mathbf{3 4}, 38,58,28,33,62, \mathbf{3 7}, 36,41,30)$

$(69,73,86,76, \mathbf{8 0}, 100,70,75,104,79, \mathbf{7 8}, 83,72)$

$29^{2} 21^{1} 16^{2} 15^{2} 13^{2} 11^{2} 4^{2} 2^{2} 1^{8}$

$s=13, r=13, k=6, d=13$

$\Delta_{0}=\{79\} \cup\{81, \ldots, 93\} \cup\{95, \ldots, 98\} \cup\{100,102,103\} \cup\{105, \ldots, 235\}$

$\left|\Delta_{0}\right|=152$; lengths of other non-trivial orbits: 43

$w_{1}=157, w_{2}=144, w_{3}=157, w_{4}=155$

$\bar{S}_{i}=29^{2} 16^{2} 15^{2} 11^{2} 1^{10}$

$W=\left[\bar{S}_{1}, \bar{S}_{2}^{2}\right]=\mathbf{1 3 1}^{1} 11^{1} 1^{10}$. 
$\underline{n=221}$. Representation $O(1) G(1) G(1) E(2) L$.

$$
\begin{aligned}
& Y_{221}= Y_{O}^{[1,7]} Y_{G}^{[8,49]} Y_{G}^{[50,91]} Y_{E}^{[92,119]} Y_{L}^{[120,221]} \\
& X_{221}= X_{O}^{[1,7]}(5,8)(6,9) X_{G}^{[8,49]}(32,50)(33,51) X_{G}^{[50,91]}(74,117)(75,118) X_{E}^{[92,119]} \\
&(101,120)(104,123) X_{L}^{[120,221]} \\
& a_{1}=20, a_{2}=21, c_{1}=62, c_{2}=63, a_{3}=22, a_{4}=23, c_{3}=64, c_{5}=66 \\
& {[\psi(X), \psi(Y)]=}(13,17,30, \mathbf{2 0}, 24,44,14,19,48, \mathbf{2 3}, 22,27,16) \\
&(55,59,72,62, \mathbf{6 6}, 86,56,61,90,65, \mathbf{6 4}, 69,58) \\
& 29^{2} 19^{1} 16^{2} 15^{2} 13^{2} 11^{2} 1^{8}
\end{aligned}
$$

$s=13, r=13, k=6, d=13$

$\Delta_{0}=\{65\} \cup\{67, \ldots, 79\} \cup\{81, \ldots, 84\} \cup\{86,88,89\} \cup\{91, \ldots, 221\}$

$\left|\Delta_{0}\right|=152$; lengths of other non-trivial orbits: 29

$w_{1}=157, w_{2}=155, w_{3}=157, w_{4}=158$

$\bar{S}_{i}=29^{2} 16^{2} 15^{2} 11^{2} 1^{10}$

$W=\left[\bar{S}_{1}, \bar{S}_{2}^{2}\right]=\mathbf{1 3 1}^{1} 11^{1} 1^{10}$.

$\underline{n=216}$. Representation $J(1) M(2) S$.

$$
\begin{aligned}
& Y_{216}=Y_{J}^{[1,72]} Y_{M}^{[73,180]} Y_{S}^{[181,216]} \\
& X_{216}=X_{J}^{[1,72]}(62,73)(63,74) X_{M}^{[73,180]}(154,194)(157,197) X_{S}^{[181,216]} \\
& a_{1}=1, a_{2}=2, c_{1}=181, c_{2}=182, a_{3}=3, a_{4}=4, c_{3}=183, c_{5}=185 \\
& {[\psi(X), \psi(Y)]=(\mathbf{1}, 5,28,31,15, \mathbf{4}, 3,9,17,26,12)} \\
& (\ldots, 190,184, \mathbf{1 8 3}, 188, \ldots, 215,181, \mathbf{1 8 5}, 213, \ldots) \\
& 13^{2} 11^{2} 8^{2} 7^{2} 6^{2} 5^{2} 1^{4} \text { (the cycle containing } 183 \text { and } 185 \text { has length 101) } \\
& s=11, r=101, d=1111 \\
& \Delta_{0}=\{76\} \cup\{78, \ldots, 81\} \cup\{83, \ldots, 87\} \cup\{89, \ldots, 92\} \cup\{94,96,97,99,101,102,103\} \\
& \cup\{105\} \cup\{107, \ldots, 114\} \cup\{116, \ldots, 120\} \cup\{125, \ldots, 134\} \cup\{136,137,138,142\} \\
& \cup\{144,146,147,148,150,152,153,160,161\} \cup\{163, \ldots, 180\} \\
& \left|\Delta_{0}\right|=76 \text {; lengths of other non-trivial orbits: } 9,9,14 \text {, and } 14 \\
& w_{1}=127, w_{2}=133, w_{3}=136, w_{4}=137 \\
& \bar{S}_{i}=13^{2} 7^{2} 6^{2} 1^{24} \\
& W=\left[\bar{S}_{1}, \bar{S}_{2}\right]=\mathbf{4 3}^{1} 5^{1} 3^{4} 1^{16} \text {. }
\end{aligned}
$$

$n=214$. Representation $G(1) G(1) E(2) L$.

$$
\begin{aligned}
& Y_{214}=Y_{G}^{[1,42]} Y_{G}^{[43,84]} Y_{E}^{[85,112]} Y_{L}^{[113,214]} \\
& X_{214}=X_{G}^{[1,42]}(25,43)(26,44) X_{G}^{[43,84]}(67,110)(68,111) X_{E}^{[85,112]}(94,113)(97,116) \\
& X_{L}^{[113,214]} \\
& a_{1}=1, a_{2}=2, c_{1}=13, c_{2}=14, a_{3}=3, a_{4}=4, c_{3}=15, c_{5}=17 \\
& {[\psi(X), \psi(Y)]=(\mathbf{1}, 5,38,31,35,40, \mathbf{4}, 3,8,33,30,36,11)} \\
& (6,10,23,13, \mathbf{1 7}, 37,7,12,41,16, \mathbf{1 5}, 20,9) 29^{2} 16^{2} 15^{2} 13^{3} 11^{2} 1^{7} \\
& s=13, r=13, k=6, d=13 \\
& \Delta_{0}=\{58\} \cup\{60, \ldots, 72\} \cup\{74, \ldots, 77\} \cup\{79,81,82\} \cup\{84, \ldots, 214\} \\
& \left|\Delta_{0}\right|=152 \text {; no other non-trivial orbits } \\
& w_{1}=157, w_{2}=156, w_{3}=157, w_{4}=155 \\
& \bar{S}_{i}=29^{2} 16^{2} 15^{2} 11^{2} 1^{10} \\
& W=\left[\bar{S}_{1}, \bar{S}_{2}^{2}\right]=\mathbf{1 3 1}^{1} 11^{1} 1^{10} \text {. }
\end{aligned}
$$


$n=212$. Representation $G(1) E(2) I(2) I(2) E$.

$$
\begin{aligned}
& Y_{212}=Y_{G}^{[1,42]} Y_{E}^{[43,70]} Y_{I}^{[71,127]} Y_{I}^{[128,184]} Y_{E}^{[185,212]} \\
& X_{212}=X_{G}^{[1,42]}(25,68)(26,69) X_{E}^{[43,70]}(52,74)(55,77) X_{I}^{[71,127]}(106,131)(109,134) \\
& X_{I}^{[128,184]}(163,194)(166,197) X_{E}^{[185,212]} \\
& a_{1}=1, a_{2}=2, c_{1}=13, c_{2}=14, a_{3}=3, a_{4}=4, c_{3}=15, c_{5}=17 \\
& {[\psi(X), \psi(Y)]=(\mathbf{1}, 5,38,31,35,40, \mathbf{4}, 3,8,33,30,36,11)} \\
& (6,10,23,13, \mathbf{1 7}, 37,7,12,41,16, \mathbf{1 5}, 20,9) \\
& \quad 23^{1} 17^{1} 12^{2} 11^{4} 10^{2} 9^{1} 8^{2} 5^{4} 2^{4} 1^{5} \\
& s=13, r=13, k=6, d=13 \\
& \Delta_{0}=\{16\} \cup\{18, \ldots, 30\} \cup\{32, \ldots, 35\} \cup\{37,39,40\} \cup\{42, \ldots, 212\} \\
& \left|\Delta_{0}\right|=192 ; \text { no other non-trivial orbits } \\
& w_{1}=212, w_{2}=185, w_{3}=211, w_{4}=209 \\
& \bar{S}_{i}=23^{1} 17^{1} 12^{2} 11^{4} 10^{2} 9^{1} 8^{2} 5^{4} 2^{4} 1^{11} \\
& W=\left[\bar{S}_{1}^{2}, \bar{S}_{2}^{3}\right]=\mathbf{9 7}^{1} 55^{1} 8^{2} 7^{1} 5^{1} 3^{2} 1^{6} .
\end{aligned}
$$

$\underline{n=208}$. Representation $E(2) M(1) J$.

$$
\begin{aligned}
& Y_{208}=Y_{E}^{[1,28]} Y_{M}^{[29,136]} Y_{J}^{[137,208]} \\
& X_{208}=X_{E}^{[1,28]}(10,110)(13,113) X_{M}^{[29,136]}(29,137)(30,138) X_{J}^{[137,208]} \\
& \begin{aligned}
& a_{1}=26, a_{2}=27, c_{1}=198, c_{2}=199, a_{3}=25, a_{4}=18, c_{3}=197, c_{5}=203 \\
& {[\psi(X), \psi(Y)]=}(3,20, \mathbf{2 6}, 16,4,8, \mathbf{1 8}, 25,23) \\
&(177,195,205, \mathbf{1 9 7}, 207,190,179,201,198, \mathbf{2 0 3}, 192) \\
& 28^{2} 14^{2} 13^{2} 11^{2} 8^{2} 7^{2} 6^{2} 5^{2} 1^{4}
\end{aligned} \\
& \begin{aligned}
& s=9, r=11, d=99 \\
& \Delta_{0}=\{1, \ldots, 15\} \cup\{17, \ldots, 24\} \cup\{28, \ldots, 155\} \cup\{157\} \cup\{161, \ldots, 170\} \cup\{172, \ldots, 174\} \\
& \cup\{176, \ldots, 179\} \cup\{181\} \cup\{186, \ldots, 189\} \cup\{191,192,194,196,200,201,202\} \\
& \cup\{204, \ldots, 208\}
\end{aligned}
\end{aligned}
$$

$\left|\Delta_{0}\right|=186$; no other non-trivial orbits

$$
\begin{aligned}
& w_{1}=1, w_{2}=28, w_{3}=1, w_{4}=2 \\
& \bar{S}_{i}=28^{2} 14^{2} 13^{2} 8^{2} 7^{2} 5^{2} 2^{6} 1^{24}
\end{aligned}
$$$$
W=\left[\bar{S}_{1}, \bar{S}_{2}^{2}\right]=113^{1} 53^{1} 1^{20} \text {. }
$$

$n=206$. Representation $O(1) G(1) G(1) E(2) I(2) F$.

$$
\begin{aligned}
& Y_{206}=Y_{O}^{[1,7]} Y_{G}^{[8,49]} Y_{G}^{[50,91]} Y_{E}^{[92,119]} Y_{I}^{[120,176]} Y_{F}^{[177,206]} \\
& X_{206}=X_{O}^{[1,7]}(5,8)(6,9) X_{G}^{[8,49]}(32,50)(33,51) X_{G}^{[50,91]}(74,117)(75,118) X_{E}^{[92,119]} \\
& (101,123)(104,126) X_{I}^{[120,176]}(155,177)(158,180) X_{F}^{[177,206]} \\
& a_{1}=20, a_{2}=21, c_{1}=62, c_{2}=63, a_{3}=22, a_{4}=23, c_{3}=64, c_{5}=66 \\
& {[\psi(X), \psi(Y)]=(13,17,30, \mathbf{2 0}, 24,44,14,19,48, \mathbf{2 3}, 22,27,16)} \\
& (55,59,72,62, \mathbf{6 6}, 86,56,61,90,65, \mathbf{6 4}, 69,58) \\
& 19^{1} 17^{1} 15^{2} 13^{2} 11^{6} 5^{2} 2^{2} 1^{8} \\
& s=13, r=13, k=6, d=13 \\
& \Delta_{0}=\{65\} \cup\{67, \ldots, 79\} \cup\{81, \ldots, 84\} \cup\{86,88,89\} \cup\{91, \ldots, 206\} \\
& \left|\Delta_{0}\right|=137 \text {; lengths of other non-trivial orbits: } 29 \\
& w_{1}=127, w_{2}=130, w_{3}=157, w_{4}=158 \\
& \bar{S}_{i}=17^{1} 15^{2} 11^{6} 5^{2} 2^{2} 1^{10} \\
& W=\left[\bar{S}_{1}, \bar{S}_{2}^{2}\right]=\mathbf{7 3}^{1} 15^{1} 10^{2} 9^{1} 5^{2} 1^{1} \text {. }
\end{aligned}
$$


$\underline{n=205}$. Representation $F(2) E(1) G(1) G(1) H(3) C$.

$$
\begin{aligned}
& \hline Y_{205}=Y_{F}^{[1,30]} Y_{E}^{[31,58]} Y_{G}^{[59,100]} Y_{G}^{[101,142]} Y_{H}^{[143,184]} Y_{C}^{[185,205]} \\
& X_{205}=X_{F}^{[1,30]}(1,40)(4,43) X_{E}^{[31,58]}(56,59)(57,60) X_{G}^{[59,100]}(83,101)(84,102) \\
& X_{G}^{[101,142]}(125,143)(126,144) X_{H}^{[143,184]}(166,192)(169,198) X_{C}^{[185,205]} \\
& a_{1}=71, a_{2}=72, c_{1}=113, c_{2}=114, a_{3}=73, a_{4}=74, c_{3}=115, c_{5}=117 \\
& {[\psi(X), \psi(Y)]=}(64,68,81,71,75,95,65,70,99, \mathbf{7 4}, 73,78,67) \\
&(106,110,123,113, \mathbf{1 1 7}, 137,107,112,141,116, \mathbf{1 1 5}, 120,109) \\
& 23^{1} 21^{1} 19^{1} 13^{2} 12^{4} 11^{2} 4^{2} 3^{1} 1^{9}
\end{aligned}
$$

$s=13, r=13, k=6, d=13$

$\Delta_{0}=\{116\} \cup\{118, \ldots, 130\} \cup\{132, \ldots, 135\} \cup\{137,139,140\} \cup\{142, \ldots, 205\}$

$\left|\Delta_{0}\right|=85$; lengths of other non-trivial orbits: 80

$w_{1}=179, w_{2}=177, w_{3}=178, w_{4}=176$

$\bar{S}_{i}=23^{1} 21^{1} 19^{1} 4^{2} 3^{1} 1^{11}$

$W=\left[\bar{S}_{1}, \bar{S}_{2}^{4}\right]=47^{1} 8^{2} 5^{1} 3^{3} 1^{8}$.

$\underline{n=201}$. Representation $C(3) N(1) J$.

$$
\begin{aligned}
& \hline Y_{201}=Y_{C}^{[1,21]} Y_{N}^{[22,129]} Y_{J}^{[130,201]} \\
& X_{201}=X_{C}^{[1,21]}(8,54)(14,56) X_{N}^{[22,129]}(22,130)(23,131) X_{J}^{[130,201]} \\
& a_{1}=1, a_{2}=2, c_{1}=191, c_{2}=192, a_{3}=3, a_{4}=4, c_{3}=190, c_{5}=196 \\
& \begin{aligned}
{[\psi(X), \psi(Y)]=} & (\mathbf{1}, 5,8,59,120,115,83,15, \mathbf{4}, 3,21,57,87,122,61,54,16) \\
& (170,188,198, \mathbf{1 9 0}, 200,183,172,194,191, \mathbf{1 9 6}, 185) \\
& 43^{1} 16^{2} 11^{2} 10^{2} 9^{2} 8^{2} 5^{2} 4^{2} 1^{4}
\end{aligned} \\
& s=17, r=11, d=187 \\
& \Delta_{0}=\{4\} \cup\{6, \ldots, 148\} \cup\{150\} \cup\{154, \ldots, 163\} \cup\{165,166,167\} \cup\{169, \ldots \\
& \cup\{174\} \cup\{179, \ldots, 182\} \cup\{184,185,187,189,193,194,195\} \cup\{197, \ldots \\
&\left|\Delta_{0}\right|=179 ; \text { no other non-trivial orbits } \\
& w_{1}=6, w_{2}=20, w_{3}=6, w_{4}=4 \\
& \bar{S}_{i}=43^{1} 16^{2} 10^{2} 9^{2} 8^{2} 5^{2} 4^{2} 1^{32} \\
& W=\left[\bar{S}_{1}, \bar{S}_{2}^{2}\right]=\mathbf{1 3 1}^{1} 13^{2} 4^{2} 1^{14} .
\end{aligned}
$$$$
a_{1}=1, a_{2}=2, c_{1}=191, c_{2}=192, a_{3}=3, a_{4}=4, c_{3}=190, c_{5}=196
$$$$
(170,188,198,190,200,183,172,194,191,196,185)
$$

$\Delta_{0}=\{4\} \cup\{6, \ldots, 148\} \cup\{150\} \cup\{154, \ldots, 163\} \cup\{165,166,167\} \cup\{169, \ldots, 172\}$

$$
\cup\{174\} \cup\{179, \ldots, 182\} \cup\{184,185,187,189,193,194,195\} \cup\{197, \ldots, 201\}
$$

$n=200$. Representation $G(1) J(1) J(1) A$.

$$
\begin{aligned}
& Y_{200}=Y_{G}^{[1,42]} Y_{J}^{[43,114]} Y_{J}^{[115,186]} Y_{A}^{[187,200]} \\
& X_{200}=X_{G}^{[1,42]}(25,43)(26,44) X_{J}^{[43,114]}(104,115)(105,116) X_{J}^{[115,186]}(176,187) \\
& (177,188) X_{A}^{[187,200]} \\
& a_{1}=1, a_{2}=2, c_{1}=13, c_{2}=14, a_{3}=3, a_{4}=4, c_{3}=15, c_{5}=17 \\
& {[\psi(X), \psi(Y)]=(\mathbf{1}, 5,38,31,35,40, \mathbf{4}, 3,8,33,30,36,11)} \\
& (6,10,23,13, \mathbf{1 7}, 37,7,12,41,16, \mathbf{1 5}, 20,9) 12^{4} 11^{6} 8^{4} 5^{4} 1^{8} \\
& s=13, r=13, k=6, d=13 \\
& \Delta_{0}=\{16\} \cup\{18, \ldots, 30\} \cup\{32, \ldots, 35\} \cup\{37,39,40\} \cup\{42, \ldots, 200\} \\
& \left|\Delta_{0}\right|=180 \text {; no other non-trivial orbits } \\
& w_{1}=18, w_{2}=19, w_{3}=18, w_{4}=16 \\
& \bar{S}_{i}=12^{4} 11^{6} 8^{4} 5^{4} 1^{14} \\
& W=\left[\bar{S}_{1}, \bar{S}_{2}^{6}\right]=\mathbf{1 0 3}^{1} 12^{2} 11^{1} 9^{3} 3^{3} 1^{6} \text {. }
\end{aligned}
$$


$n=198$. Representation $G(1) G(1) E(2) E(1) E(2) F$.

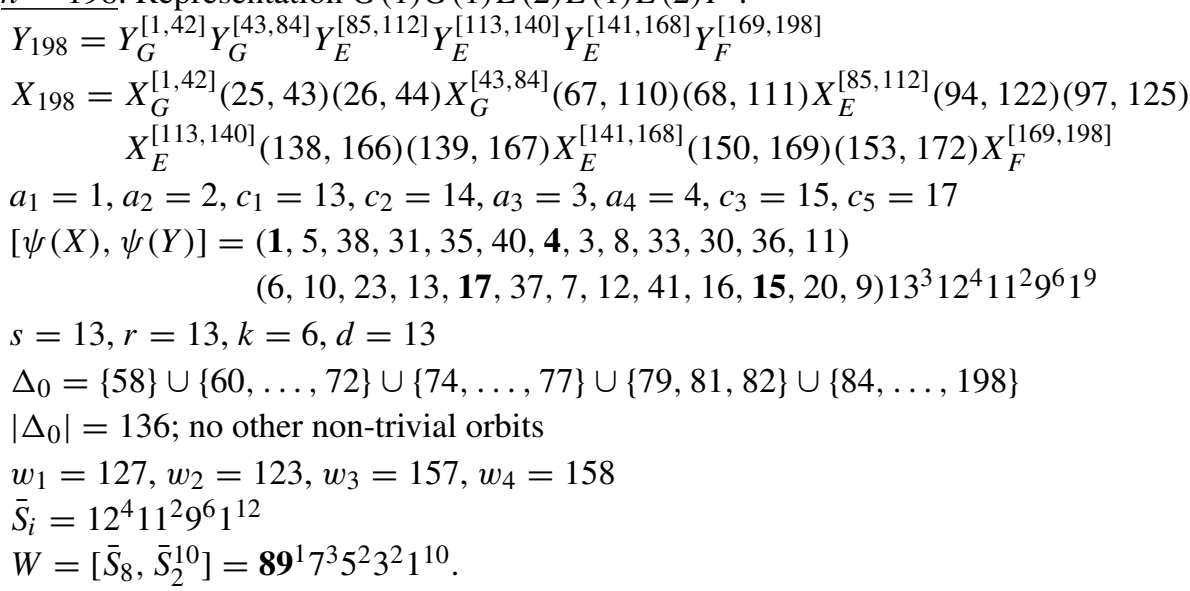

$n=193$. Representation $G(1) J(1) J(1) O$.

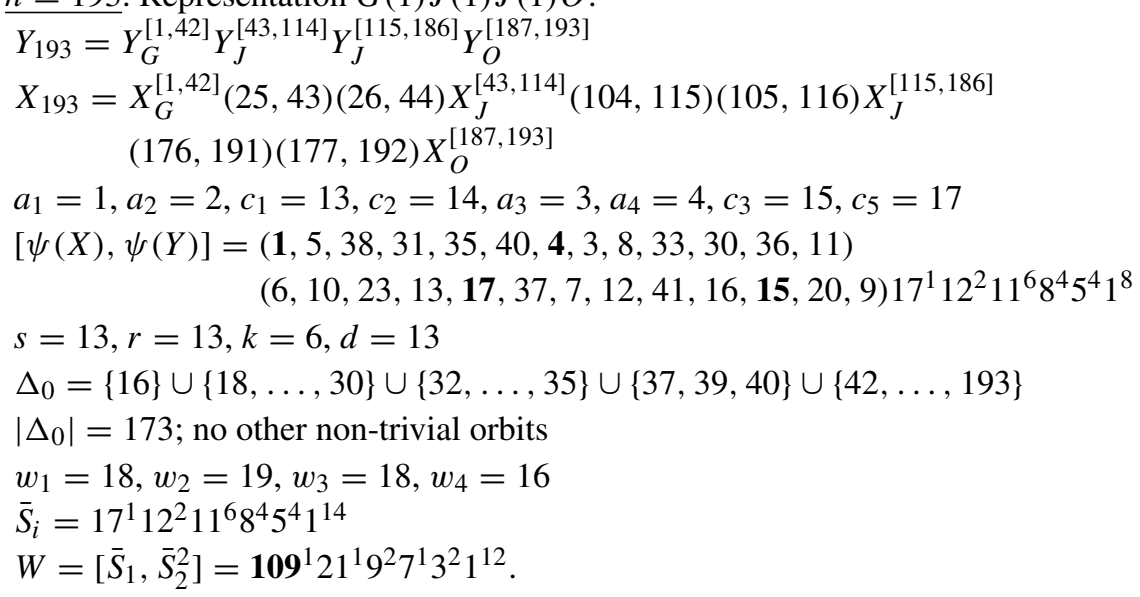

$n=192$. Representation $G(1) G(1) M$.

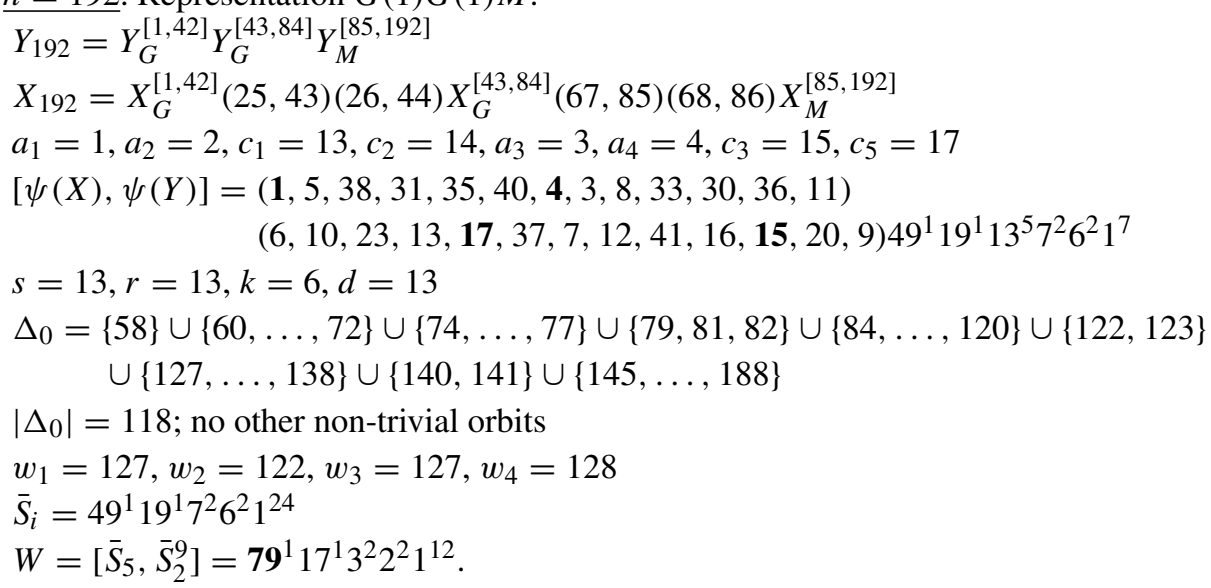


$\underline{n=191}$. Representation $A(1) G(1) G(1) J(1) C$.

$$
\begin{aligned}
& \hline Y_{191}= Y_{A}^{[1,14]} Y_{G}^{[15,56]} Y_{G}^{[57,98]} Y_{J}^{[99,170]} Y_{C}^{[171,191]} \\
& X_{191}= X_{A}^{[1,14]}(1,15)(2,16) X_{G}^{[15,56]}(39,57)(40,58) X_{G}^{[57,98]}(81,99)(82,100) X_{J}^{[99,170]} \\
&(160,171)(161,172) X_{C}^{[171,191]} \\
& a_{1}=27, a_{2}=28, c_{1}=69, c_{2}=70, a_{3}=29, a_{4}=30, c_{3}=71, c_{5}=73 \\
& {[\psi(X), \psi(Y)]=}(20,24,37, \mathbf{2 7}, 31,51,21,26,55, \mathbf{3 0}, 29,34,23) \\
&(62,66,79,69, \mathbf{7 3}, 93,63,68,97,72, \mathbf{7 1}, 76,65) \\
& 19^{1} 13^{4} 12^{2} 11^{2} 8^{2} 5^{2} 4^{2} 2^{2} 1^{10} \\
& s=13, r=13, k=6, d=13 \\
& \Delta_{0}=\{72\} \cup\{74, \ldots, 86\} \cup\{88, \ldots, 91\} \cup\{93,95,96\} \cup\{98, \ldots, 191\} \\
&\left|\Delta_{0}\right|=115 ; \text { no other non-trivial orbits } \\
& w_{1}=127, w_{2}=137, w_{3}=127, w_{4}=128 \\
& \bar{S}_{i}=19^{1} 12^{2} 11^{2} 8^{2} 5^{2} 4^{2} 2^{2} 1^{12} \\
& W=\left[\bar{S}_{1}, \bar{S}_{2}^{8}\right]=\mathbf{5 9}^{1} 29^{1} 5^{1} 3^{1} 1^{19} .
\end{aligned}
$$

$n=185$. Representation $J(1) E(2) I(2) E$.

$$
\begin{aligned}
& \hline Y_{185}= Y_{J}^{[1,72]} Y_{E}^{[73,100]} Y_{I}^{[101,157]} Y_{E}^{[158,185]} \\
& X_{185}= X_{J}^{[1,72]}(62,98)(63,99) X_{E}^{[73,100]}(82,104)(85,107) X_{I}^{[101,157]} \\
&(136,167)(139,170) X_{E}^{[158,185]} \\
& a_{1}=1, a_{2}=2, c_{1}=183, c_{2}=184, a_{3}=3, a_{4}=4, c_{3}=182, c_{5}=173 \\
& {[\psi(X), \psi(Y)]=(\mathbf{1}, 5,28,31,15, \mathbf{4}, 3,9,17,26,12) } \\
& \quad(160,177,183, \mathbf{1 7 3}, 161,165,175, \mathbf{1 8 2}, 180) 17^{1} 12^{2} 11^{4} 10^{2} 8^{4} 5^{4} 2^{2} 1^{4} \\
& s=11, r=9, d=99 \\
& \Delta_{0}=\{4\} \cup\{6, \ldots, 14\} \cup\{16,18,19,21,25,26,28\} \cup\{30, \ldots, 34\} \cup\{36,37,38\} \\
& \cup\{40, \ldots, 45\} \cup\{50, \ldots, 90\} \cup\{92,93\} \cup\{95, \ldots, 153\} \cup\{156, \ldots, 172\} \\
& \cup\{174, \ldots, 181\} \cup\{185\}
\end{aligned}
$$

$\left|\Delta_{0}\right|=159$; no other non-trivial orbits

$$
\begin{aligned}
& w_{1}=157, w_{2}=153, w_{3}=158, w_{4}=159 \\
& \bar{S}_{i}=17^{1} 10^{2} 8^{4} 5^{4} 4^{6} 2^{2} 1^{42} \\
& W=\left[\bar{S}_{1}^{2},\left[\bar{S}_{1}^{3}, \bar{S}_{2}^{3}\right]\right]=\mathbf{9 7}^{1} 9^{1} 7^{1} 5^{1} 3^{1} 2^{2} 1^{34} .
\end{aligned}
$$

$n=180$. Representation $G(1) M(2) F$.

$$
\begin{aligned}
& Y_{180}=Y_{G}^{[1,42]} Y_{M}^{[43,150]} Y_{F}^{[151,180]} \\
& X_{180}=X_{G}^{[1,42]}(25,43)(26,44) X_{M}^{[43,150]}(124,151)(127,154) X_{F}^{[151,180]} \\
& a_{1}=1, a_{2}=2, c_{1}=13, c_{2}=14, a_{3}=3, a_{4}=4, c_{3}=15, c_{5}=17 \\
& {[\psi(X), \psi(Y)]=(\mathbf{1}, 5,38,31,35,40, \mathbf{4}, 3,8,33,30,36,11)} \\
& \quad(6,10,23,13, \mathbf{1 7}, 37,7,12,41,16, \mathbf{1 5}, 20,9) 32^{2} 17^{2} 13^{2} 7 \\
& s=13, r=13, k=6, d=13 \\
& \Delta_{0}=\{16\} \cup\{18, \ldots, 30\} \cup\{32, \ldots, 35\} \cup\{37,39,40\} \cup\{42, \ldots, 78\} \\
& \quad \cup\{80,81\} \cup\{85, \ldots, 96\} \cup\{98,99\} \cup\{103, \ldots, 146\} \cup\{151, \ldots \\
& \left|\Delta_{0}\right|=148 ; \text { no other non-trivial orbits } \\
& w_{1}=157, w_{2}=155, w_{3}=157, w_{4}=158 \\
& \bar{S}_{i}=32^{2} 17^{2} 7^{2} 6^{2} 1^{24} \\
& W=\left[\bar{S}_{1}, \bar{S}_{2}^{6}\right]=\mathbf{8 3}^{1} 41^{1} 3^{2} 1^{18} .
\end{aligned}
$$$$
a_{1}=1, a_{2}=2, c_{1}=13, c_{2}=14, a_{3}=3, a_{4}=4, c_{3}=15, c_{5}=17
$$$$
(6,10,23,13, \mathbf{1 7}, 37,7,12,41,16, \mathbf{1 5}, 20,9) 32^{2} 17^{2} 13^{2} 7^{2} 6^{2} 1^{4}
$$$$
\cup\{80,81\} \cup\{85, \ldots, 96\} \cup\{98,99\} \cup\{103, \ldots, 146\} \cup\{151, \ldots, 180\}
$$ 
$\underline{n=172}$. Representation $G(1) E(2) L$.

$Y_{172}=Y_{G}^{[1,42]} Y_{E}^{[43,70]} Y_{L}^{[71,172]}$
$X_{172}=X_{G}^{[1,42]}(25,68)(26,69) X_{E}^{[43,70]}(52,71)(55,74) X_{L}^{[71,172]}$

$a_{1}=1, a_{2}=2, c_{1}=13, c_{2}=14, a_{3}=3, a_{4}=4, c_{3}=15, c_{5}=17$

$[\psi(X), \psi(Y)]=(\mathbf{1}, 5,38,31,35,40, \mathbf{4}, 3,8,33,30,36,11)$

$(6,10,23,13, \mathbf{1 7}, 37,7,12,41,16, \mathbf{1 5}, 20,9) 29^{2} 16^{2} 15^{2} 11^{2} 1^{4}$

$s=13, r=13, k=6, d=13$

$\Delta_{0}=\{16\} \cup\{18, \ldots, 30\} \cup\{32, \ldots, 35\} \cup\{37,39,40\} \cup\{42, \ldots, 172\}$

$\left|\Delta_{0}\right|=152$; no other non-trivial orbits

$w_{1}=157, w_{2}=158, w_{3}=157, w_{4}=155$

$\bar{S}_{i}=29^{2} 16^{2} 15^{2} 11^{2} 1^{10}$

$W=\left[\bar{S}_{1}, \bar{S}_{2}^{2}\right]=\mathbf{1 3 1}^{1} 11^{1} 1^{10}$.

$\underline{n=171}$. Representation $G(1) J(1) H(3) B$.

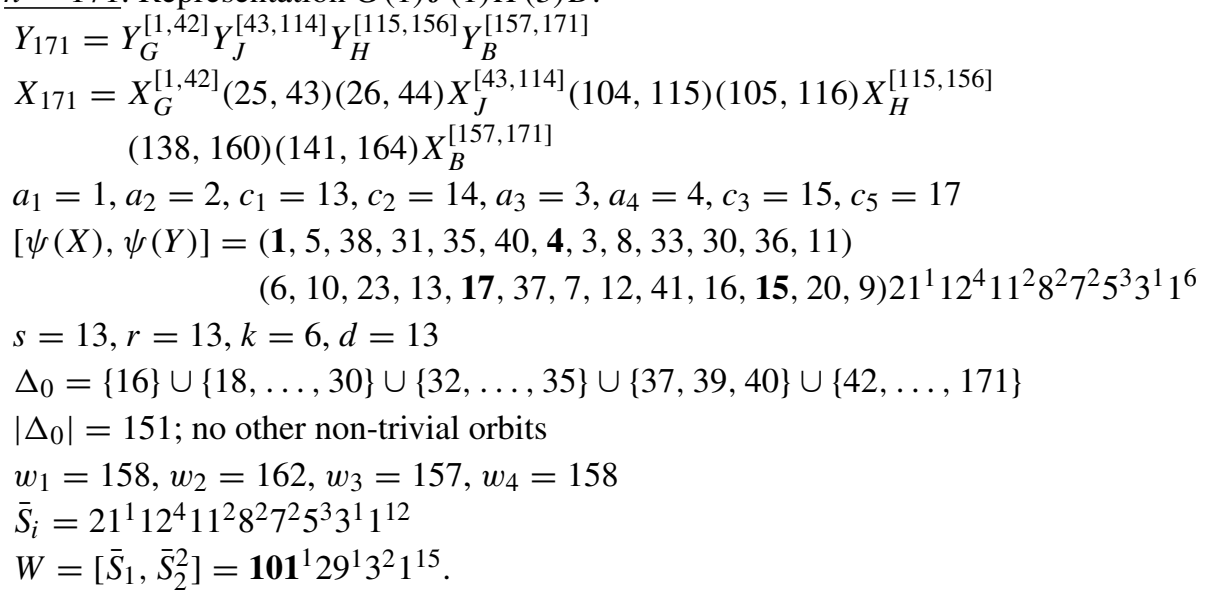

$\underline{n=170}$. Representation $G(1) J(1) E(2) E$.

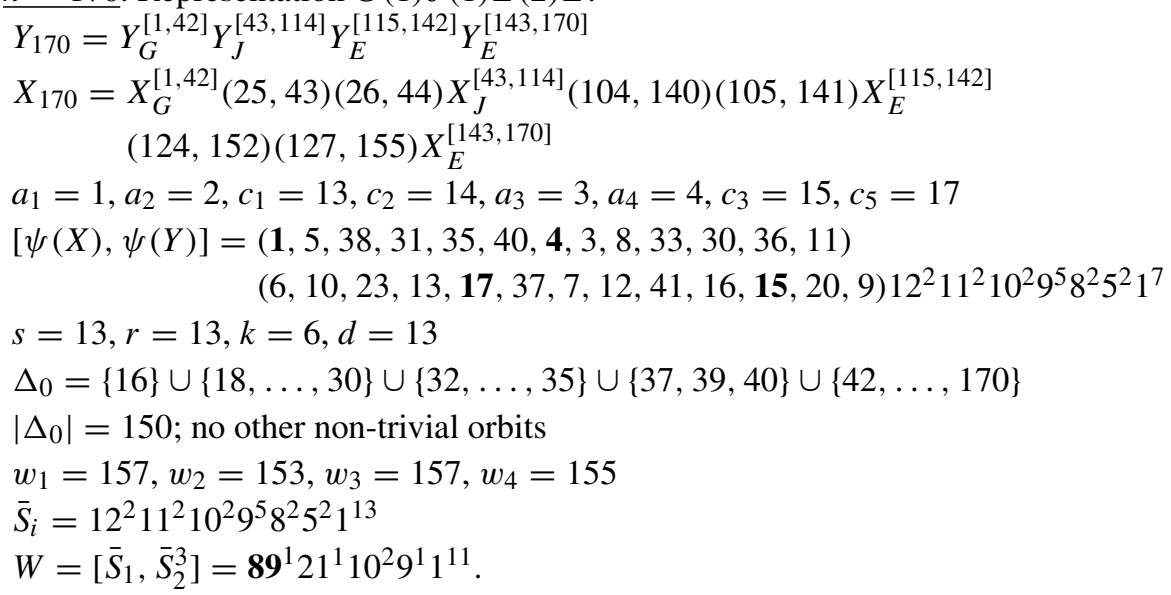


$n=165$. Representation $G(1) N(3) B$.

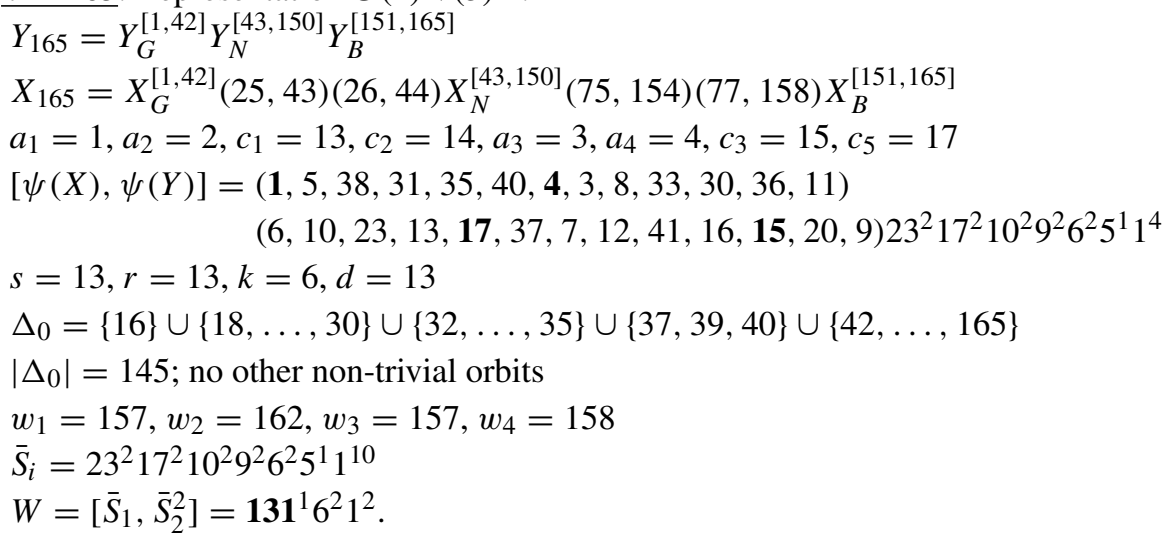

$n=164$. Representation $G(1) J(1) E(2) D$.

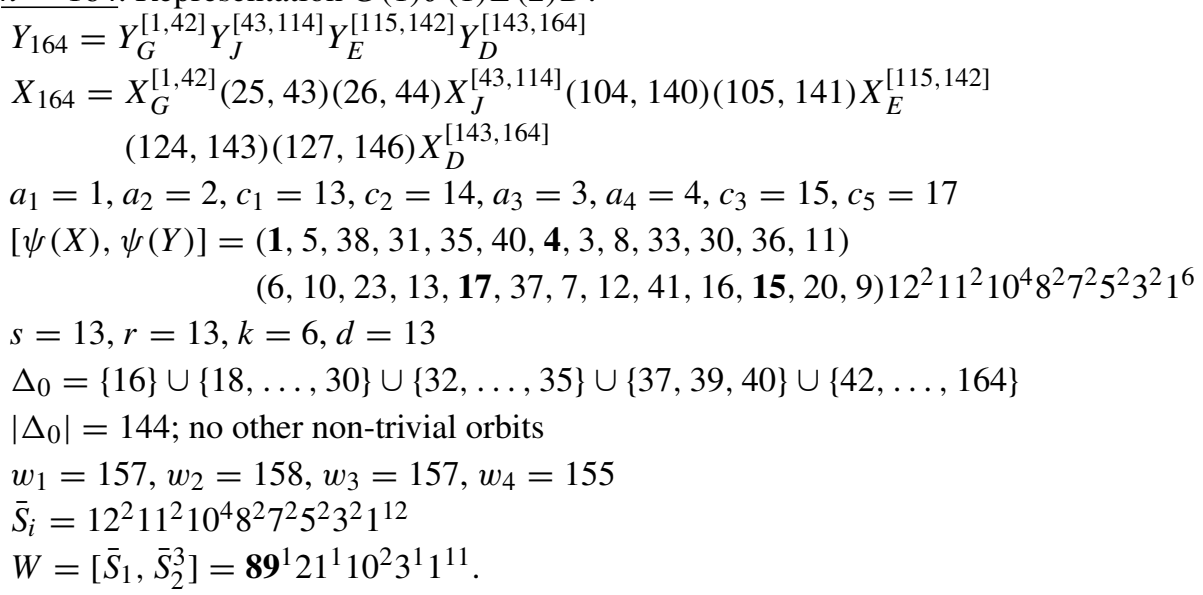

$\underline{n=163}$. Representation $C(1) G(1) G(1) E(2) F$.

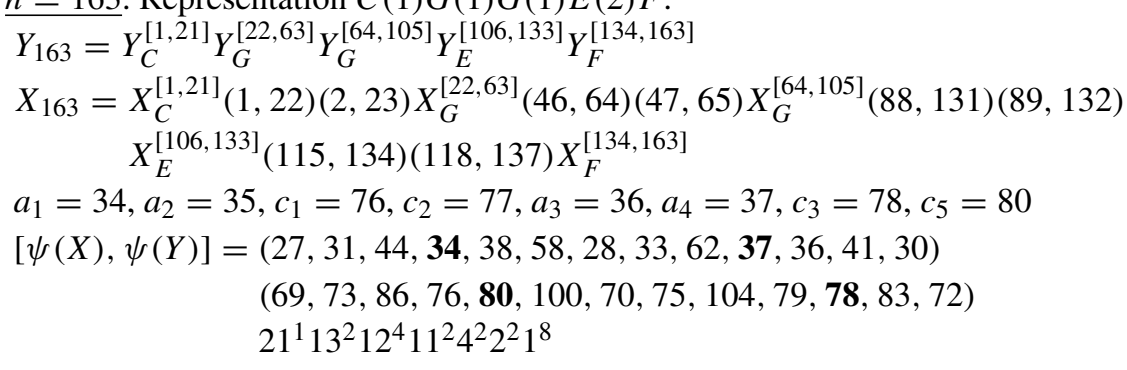

$s=13, r=13, k=6, d=13$

$\Delta_{0}=\{79\} \cup\{81, \ldots, 93\} \cup\{95, \ldots, 98\} \cup\{100,102,103\} \cup\{105, \ldots, 163\}$

$\left|\Delta_{0}\right|=80$; lengths of other non-trivial orbits: 43

$$
\begin{aligned}
& w_{1}=134, w_{2}=115, w_{3}=89, w_{4}=90 \\
& \bar{S}_{i}=12^{4} 11^{2} 1^{10} \\
& W=\left[\bar{S}_{1}^{4}, \bar{S}_{2}^{6}\right]=\mathbf{5 3}^{1} 9^{1} 4^{2} 1^{10} .
\end{aligned}
$$


$\underline{n=156}$. Representation $A(1) G(1) G(1) E(2) F$.

$$
\begin{aligned}
& Y_{156}=Y_{A}^{[1,14]} Y_{G}^{[15,56]} Y_{G}^{[57,98]} Y_{E}^{[99,126]} Y_{F}^{[127,156]} \\
& X_{156}=X_{A}^{[1,14]}(1,15)(2,16) X_{G}^{[15,56]}(39,57)(40,58) X_{G}^{[57,98]}(81,124)(82,125) X_{E}^{[99,126]} \\
& (108,127)(111,130) X_{F}^{[127,156]} \\
& a_{1}=27, a_{2}=28, c_{1}=69, c_{2}=70, a_{3}=29, a_{4}=30, c_{3}=71, c_{5}=73 \\
& {[\psi(X), \psi(Y)]=(20,24,37,27,31,51,21,26,55, \mathbf{3 0}, 29,34,23)} \\
& (62,66,79,69,73,93,63,68,97,72,71,76,65) \\
& 13^{4} 12^{4} 11^{2} 1^{8} \\
& s=13, r=13, k=6, d=13 \\
& \Delta_{0}=\{72\} \cup\{74, \ldots, 86\} \cup\{88, \ldots, 91\} \cup\{93,95,96\} \cup\{98, \ldots, 156\} \\
& \left|\Delta_{0}\right|=80 \text {; no other non-trivial orbits } \\
& w_{1}=89, w_{2}=91, w_{3}=90, w_{4}=91 \\
& \bar{S}_{i}=12^{4} 11^{2} 1^{10} \\
& W=\left[\bar{S}_{1}^{4}, \bar{S}_{2}^{6}\right]=\mathbf{5 3}^{1} 9^{1} 4^{2} 1^{10} \text {. }
\end{aligned}
$$

$n=150$. Representation $G(1) J(1) C(3) B$.

$$
\begin{aligned}
& Y_{150}=Y_{G}^{[1,42]} Y_{J}^{[43,114]} Y_{C}^{[115,135]} Y_{B}^{[136,150]} \\
& X_{150}=X_{G}^{[1,42]}(25,43)(26,44) X_{J}^{[43,114]}(104,115)(105,116) X_{C}^{[115,135]} \\
& (122,139)(128,143) X_{B}^{[136,150]} \\
& a_{1}=1, a_{2}=2, c_{1}=13, c_{2}=14, a_{3}=3, a_{4}=4, c_{3}=15, c_{5}=17 \\
& {[\psi(X), \psi(Y)]=(\mathbf{1}, 5,38,31,35,40, \mathbf{4}, 3,8,33,30,36,11)} \\
& (6,10,23,13,17,37,7,12,41,16,15,20,9) 12^{2} 11^{5} 8^{2} 5^{3} 4^{2} 1^{6} \\
& s=13, r=13, k=6, d=13 \\
& \Delta_{0}=\{16\} \cup\{18, \ldots, 30\} \cup\{32, \ldots, 35\} \cup\{37,39,40\} \cup\{42, \ldots, 150\} \\
& \left|\Delta_{0}\right|=130 \text {; no other non-trivial orbits } \\
& w_{1}=127, w_{2}=125, w_{3}=127, w_{4}=128 \\
& \bar{S}_{i}=12^{2} 11^{5} 8^{2} 5^{3} 4^{2} 1^{12} \\
& W=\left[\bar{S}_{1}, \bar{S}_{2}^{6}\right]=\mathbf{7 1}^{1} 12^{2} 11^{1} 9^{1} 3^{3} 1^{6} \text {. }
\end{aligned}
$$

$n=149$. Representation $O(1) G(1) G(1) E(2) F$.

$$
\begin{gathered}
Y_{149}=Y_{O}^{[1,7]} Y_{G}^{[8,49]} Y_{G}^{[59,91]} Y_{E}^{[92,119]} Y_{F}^{[120,149]} \\
X_{149}=X_{O}^{[1,7]}(5,8)(6,9) X_{G}^{[8,49]}(32,50)(33,51) X_{G}^{[59,91]}(74,117)(75,118) X_{E}^{[92,119]} \\
(101,120)(104,123) X_{F}^{[120,149]} \\
a_{1}=20, a_{2}=21, c_{1}=62, c_{2}=63, a_{3}=22, a_{4}=23, c_{3}=64, c_{5}=66 \\
{[\psi(X), \psi(Y)]=(13,17,30, \mathbf{2 0}, 24,44,14,19,48, \mathbf{2 3}, 22,27,16)} \\
(55,59,72,62, \mathbf{6 6}, 86,56,61,90,65, \mathbf{6 4}, 69,58) \\
19^{1} 13^{2} 12^{4} 11^{2} 1^{8} \\
s=13, r=13, k=6, d=13
\end{gathered}
$$$$
\Delta_{0}=\{65\} \cup\{67, \ldots, 79\} \cup\{81, \ldots, 84\} \cup\{86,88,89\} \cup\{91, \ldots, 149\}
$$$$
\left|\Delta_{0}\right|=80 \text {; lengths of other non-trivial orbits: } 29
$$$$
w_{1}=91, w_{2}=81, w_{3}=91, w_{4}=89
$$$$
\bar{S}_{i}=12^{4} 11^{2} 1^{10}
$$$$
W=\left[\bar{S}_{1}^{4}, \bar{S}_{2}^{6}\right]=\mathbf{5 3}^{1} 9^{1} 4^{2} 1^{10} \text {. }
$$ 
$\underline{n=147}$. Representation $G(1) G(1) G(1) C$.

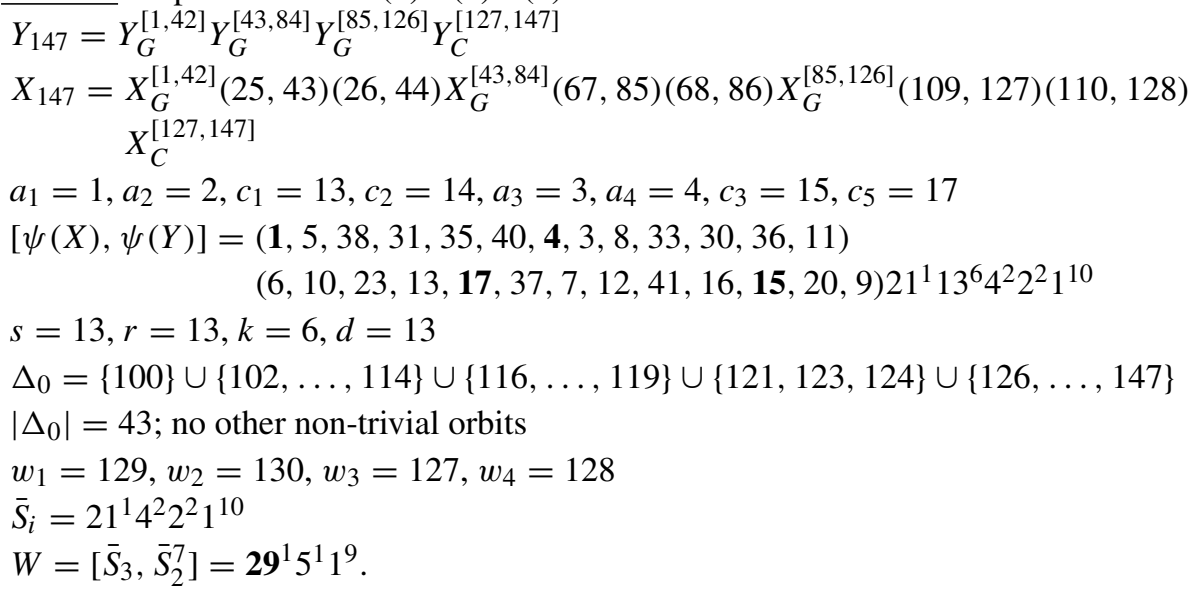

$\underline{n=142}$. Representation $G(1) G(1) E(2) F$.

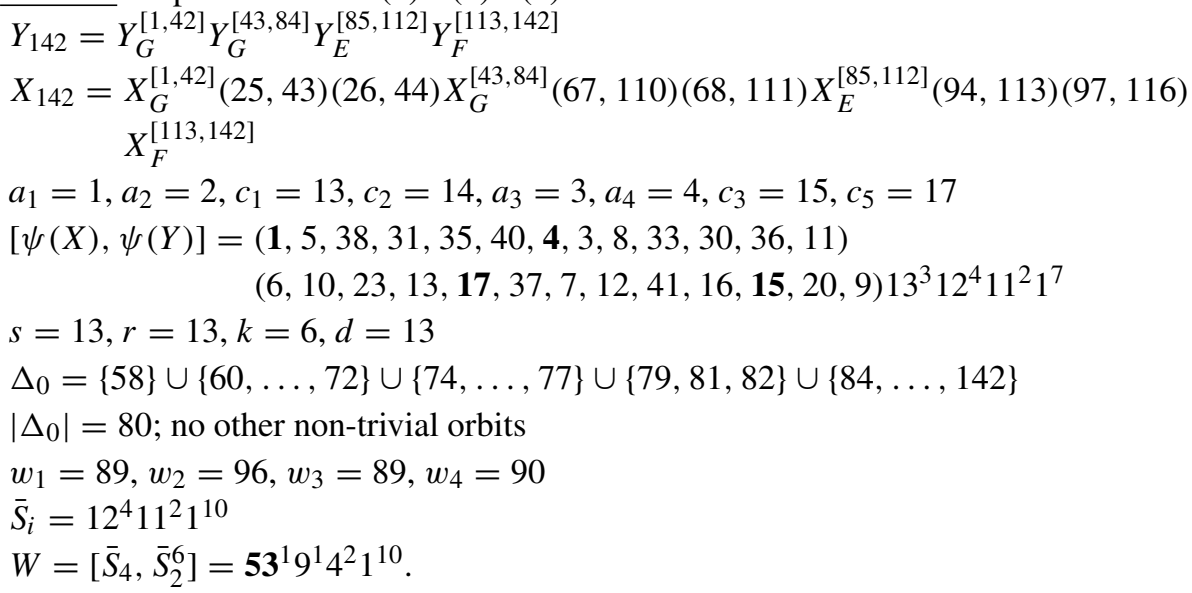

$\underline{n=136}$. Representation $G(1) J(1) R$.

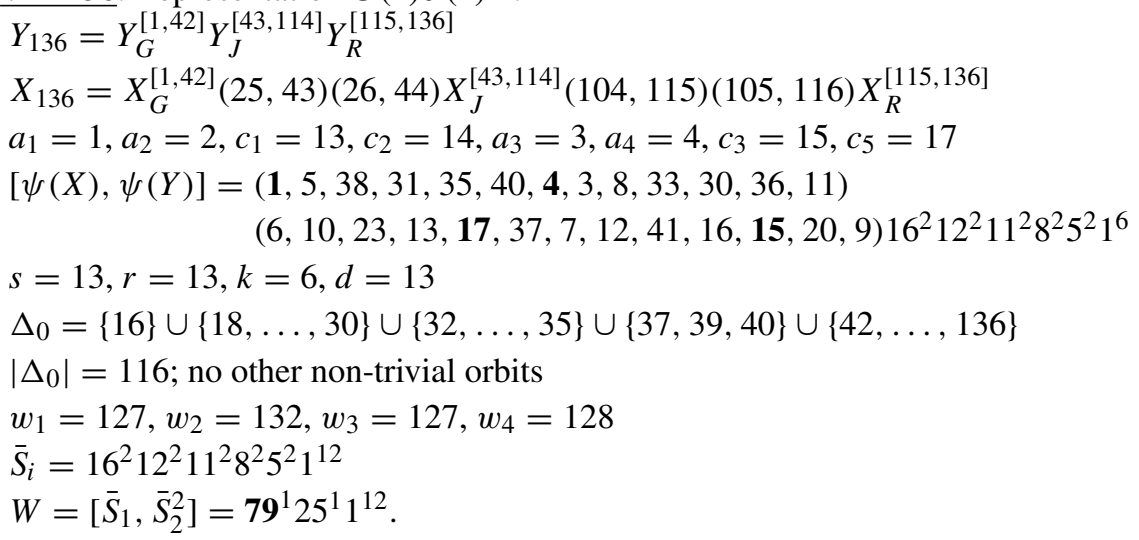


$\underline{n=135}$. Representation $G(1) J(1) Q$.

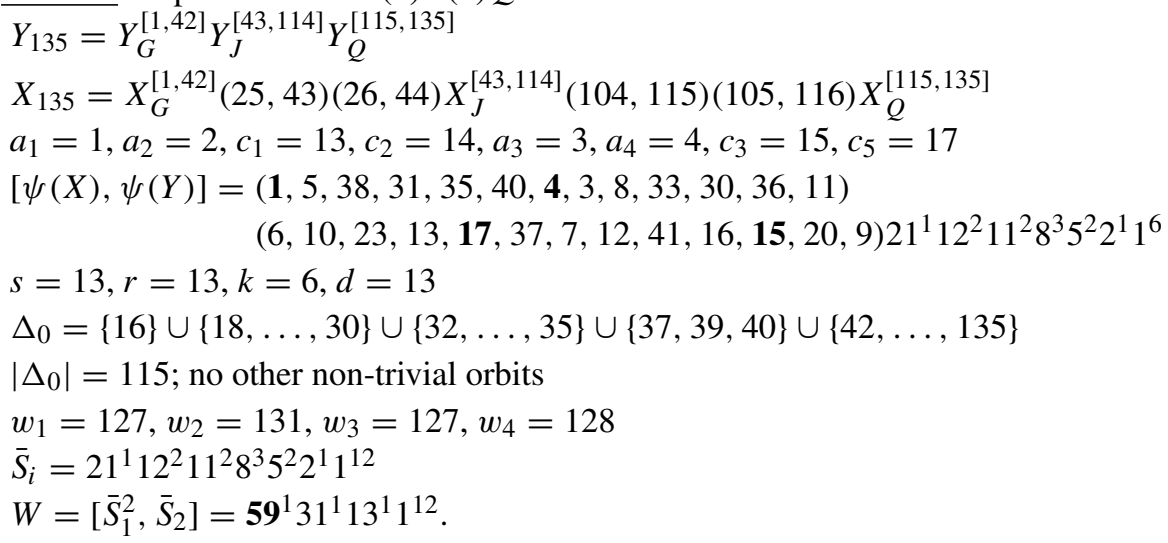

$\underline{n=133}$. Representation $G(1) G(1) G(1) O$.

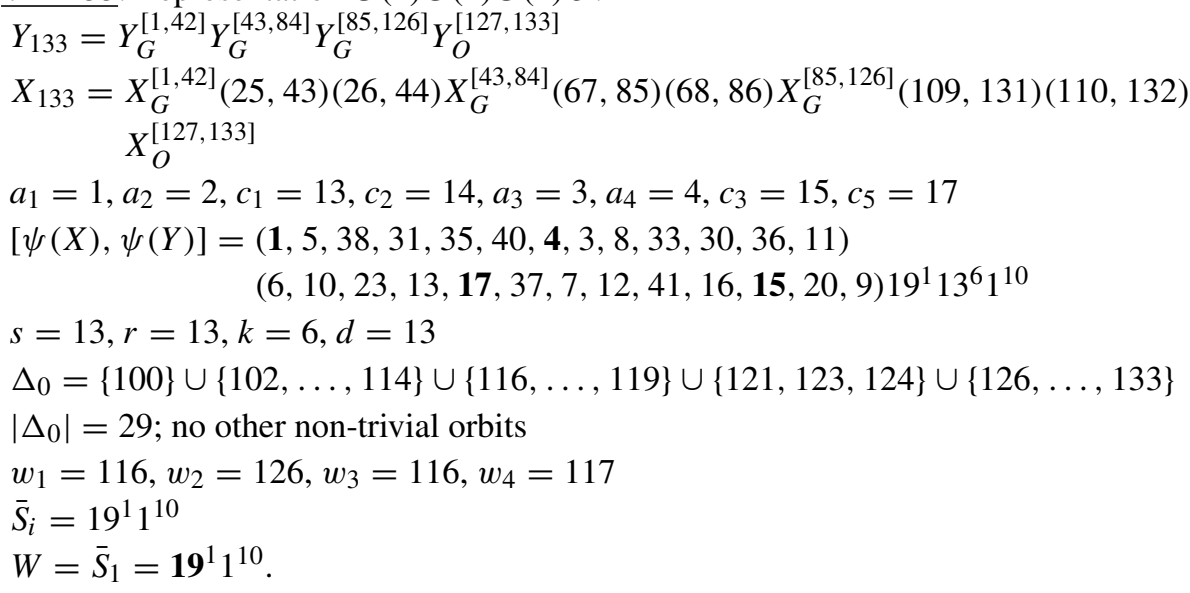

$n=129$. Representation $G(1) J(1) P$.

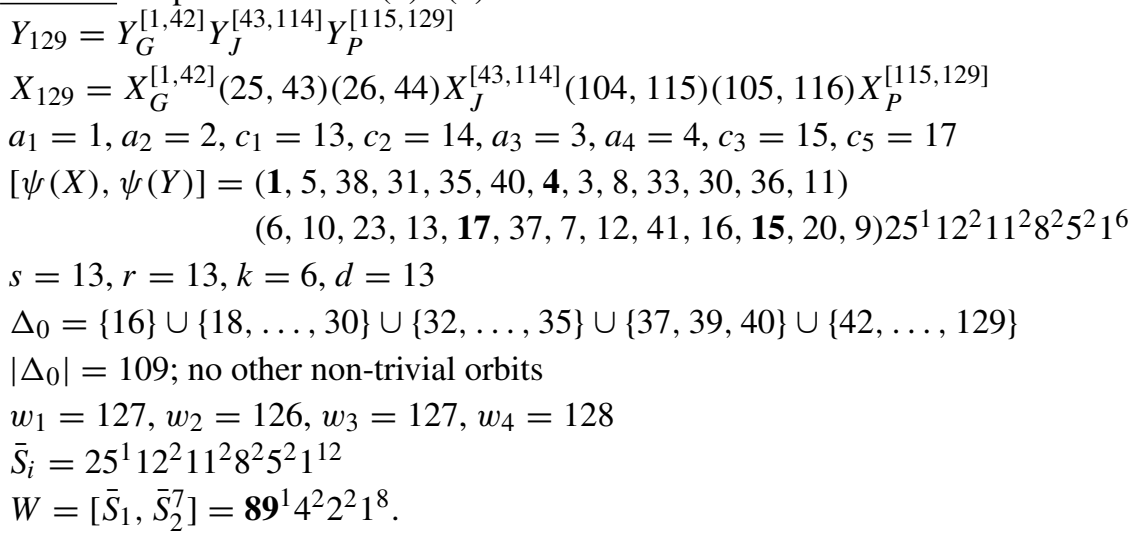


$\underline{n=128}$. Representation $G(1) J(1) A$.

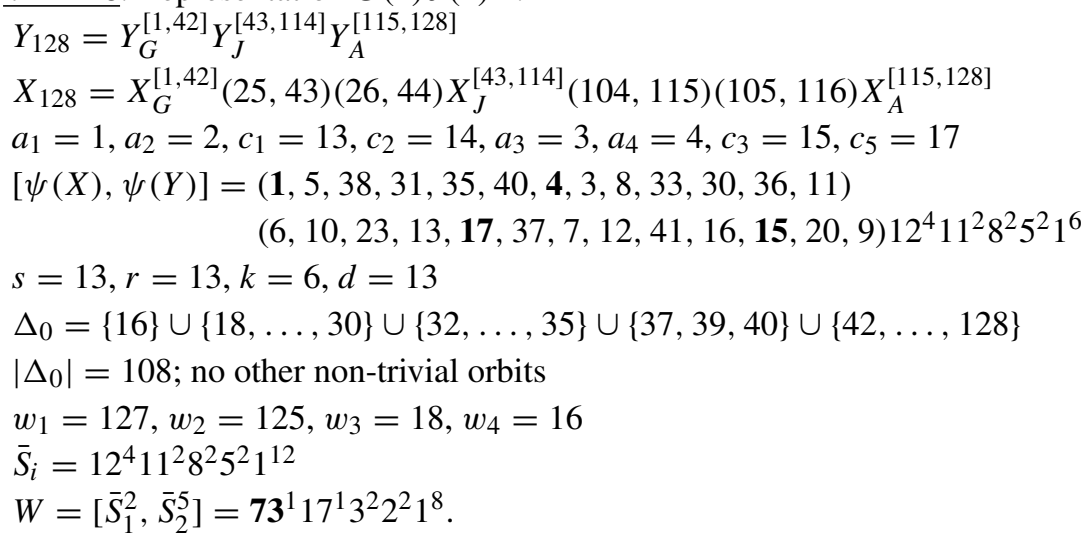

$n=127$. Representation $O(1) G(1) G(1) C(3) B$.

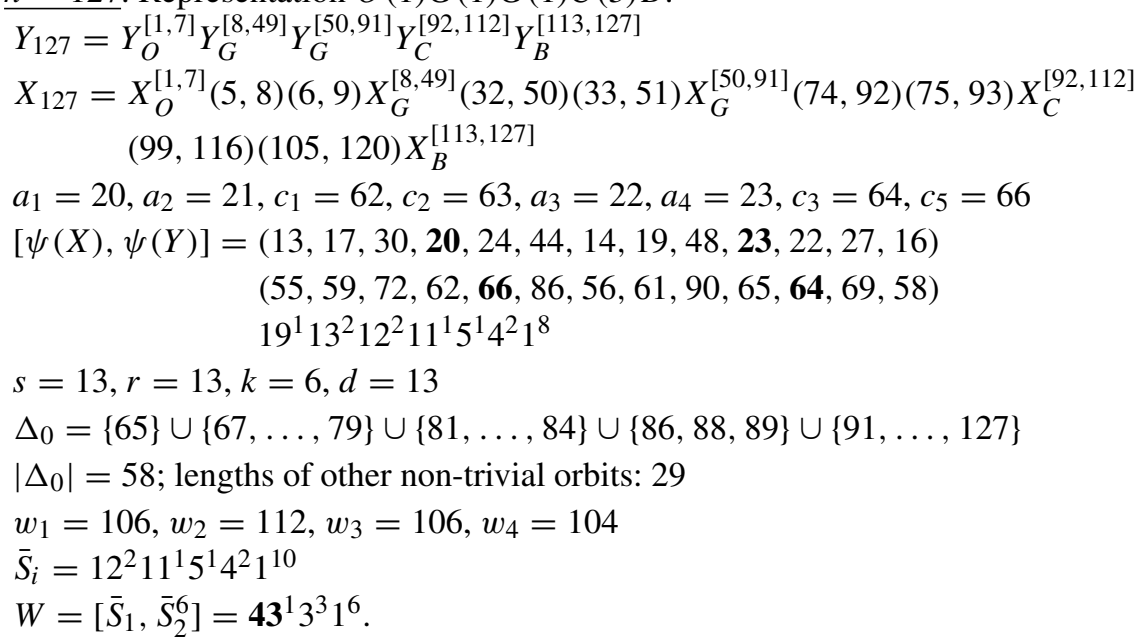

$\underline{n=121}$. Representation $G(1) J(1) O$.

$$
\begin{aligned}
& Y_{121}=Y_{G}^{[1,42]} Y_{J}^{[43,114]} Y_{O}^{[115,121]} \\
& X_{121}=X_{G}^{[1,42]}(25,43)(26,44) X_{J}^{[43,114]}(104,119)(105,120) X_{O}^{[115,121]} \\
& {[\psi(X), \psi(Y)]=(\mathbf{1}, 5,38,31,35,40, \mathbf{4}, 3,8,33,30,36,11)} \\
& s=13, r=13, k=6, d=13 \\
& \Delta_{0}=\{16\} \cup\{18, \ldots, 30\} \cup\{32, \ldots, 35\} \cup\{37,39,40\} \cup\{42, \ldots, 121\} \\
& \left|\Delta_{0}\right|=101 \text {; no other non-trivial orbits } \\
& w_{1}=18, w_{2}=19, w_{3}=18, w_{4}=16 \\
& \bar{S}_{i}=17^{1} 12^{2} 11^{2} 8^{2} 5^{2} 1^{12} \\
& W=\left[\bar{S}_{1}, \bar{S}_{2}^{6}\right]=\mathbf{8 9}^{1} 3^{2} 1^{6} \text {. }
\end{aligned}
$$$$
a_{1}=1, a_{2}=2, c_{1}=13, c_{2}=14, a_{3}=3, a_{4}=4, c_{3}=15, c_{5}=17
$$$$
(6,10,23,13,17,37,7,12,41,16,15,20,9) 17^{1} 13^{2} 12^{2} 11^{2} 8^{2} 5^{2} 1^{6}
$$ 
$\underline{n=114}$. Representation $G(1) J$.

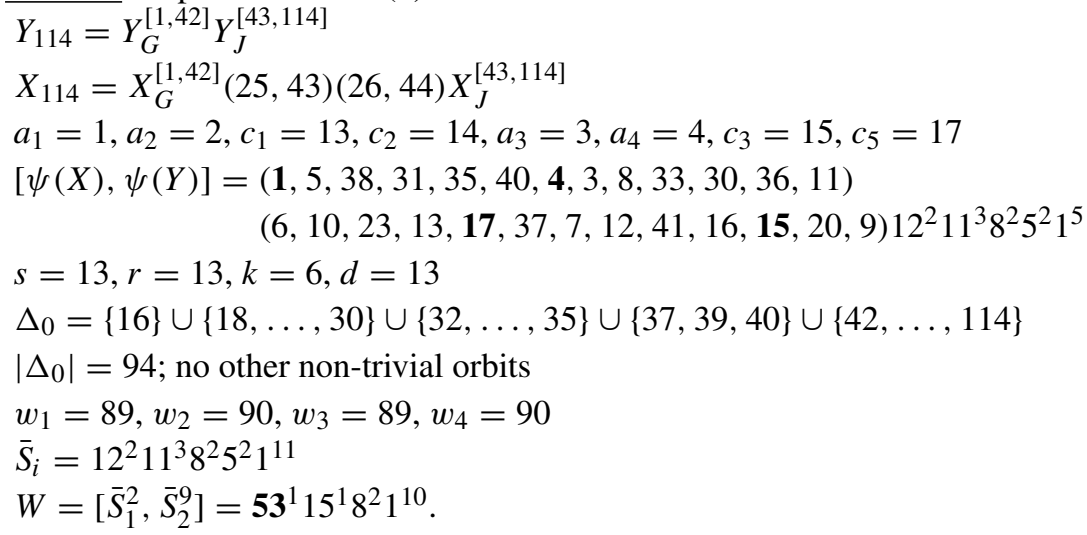

$\underline{n=113}$. Representation $R(1) G(1) G(1) O$

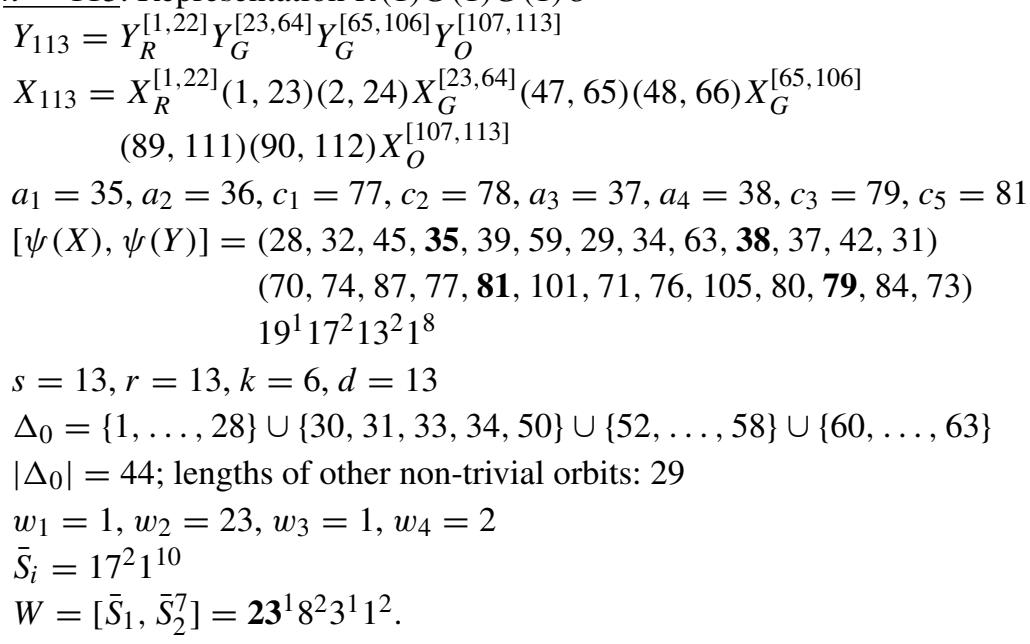

$\underline{n=112}$. Representation $G(1) H(3) C(1) O$.

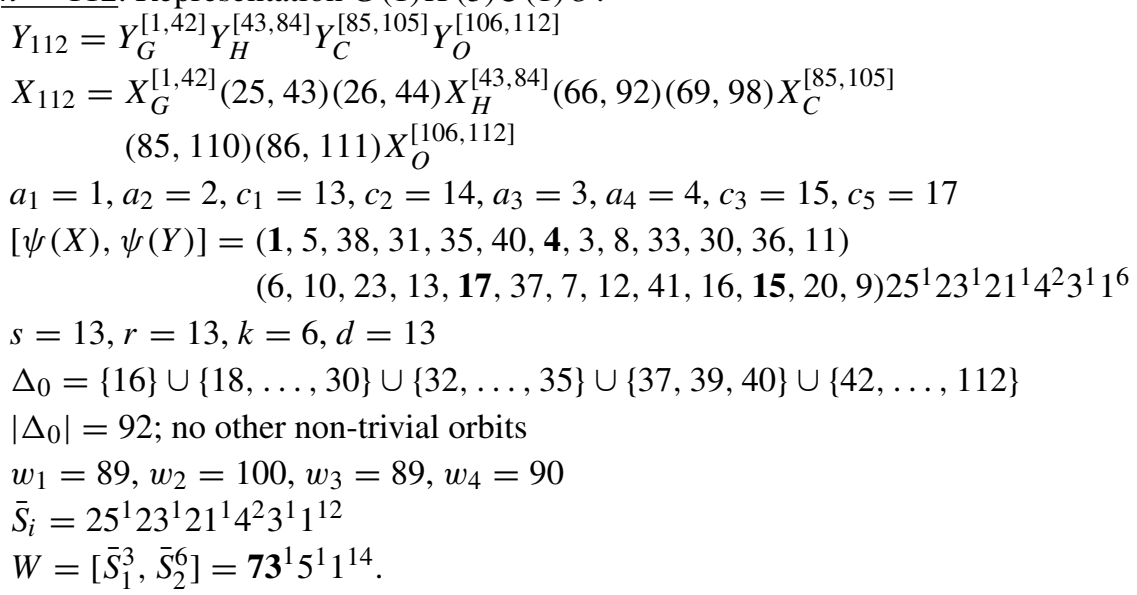


$\underline{n=108}$. Representation $G(1) T$.

$Y_{108}=Y_{G}^{[1,42]} Y_{T}^{[43,108]}$
$X_{108}=X_{G}^{[1,42]}(25,43)(26,44) X_{T}^{[43,108]}$

$a_{1}=1, a_{2}=2, c_{1}=13, c_{2}=14, a_{3}=3, a_{4}=4, c_{3}=15, c_{5}=17$

$[\psi(X), \psi(Y)]=(\mathbf{1}, 5,38,31,35,40, \mathbf{4}, 3,8,33,30,36,11)$

$(6,10,23,13,17,37,7,12,41,16,15,20,9) 39^{2} 1^{4}$

$s=13, r=13, k=6, d=13$

$\Delta_{0}=\{16\} \cup\{18, \ldots, 30\} \cup\{32, \ldots, 35\} \cup\{37,39,40\} \cup\{42, \ldots, 108\}$

$\left|\Delta_{0}\right|=88$; no other non-trivial orbits

$w_{1}=89, w_{2}=91, w_{3}=89, w_{4}=90$

$\bar{S}_{i}=3^{26} 1^{10}$

$W=\left(\bar{S}_{1} \bar{S}_{2}\right)^{2}\left[\bar{S}_{1}, \bar{S}_{2}\right]=\mathbf{7 1}^{1} 12^{1} 3^{1} 2^{1}$.

$n=106$. Representation $G(1) G(1) R$.

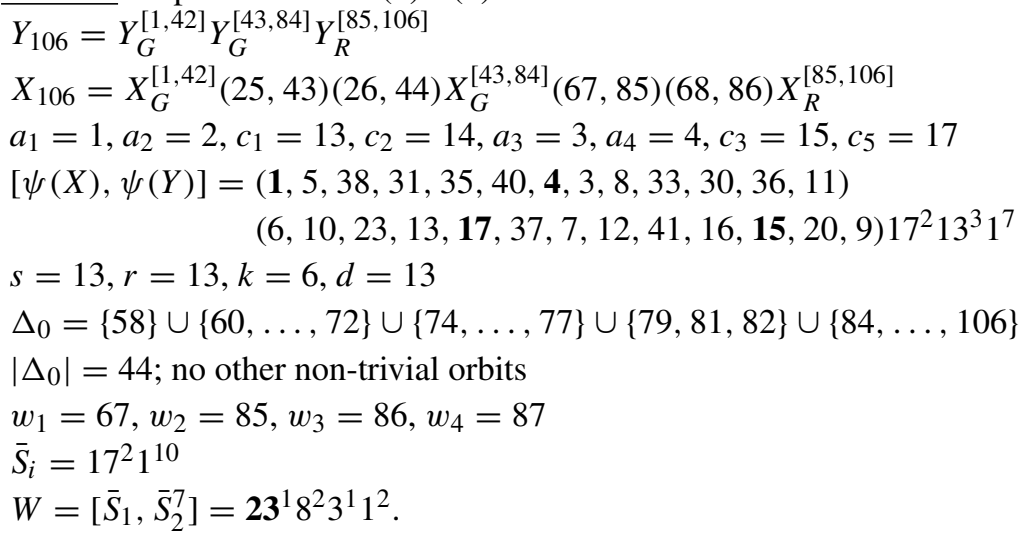

$n=105$. Representation $G(1) H(3) C$.

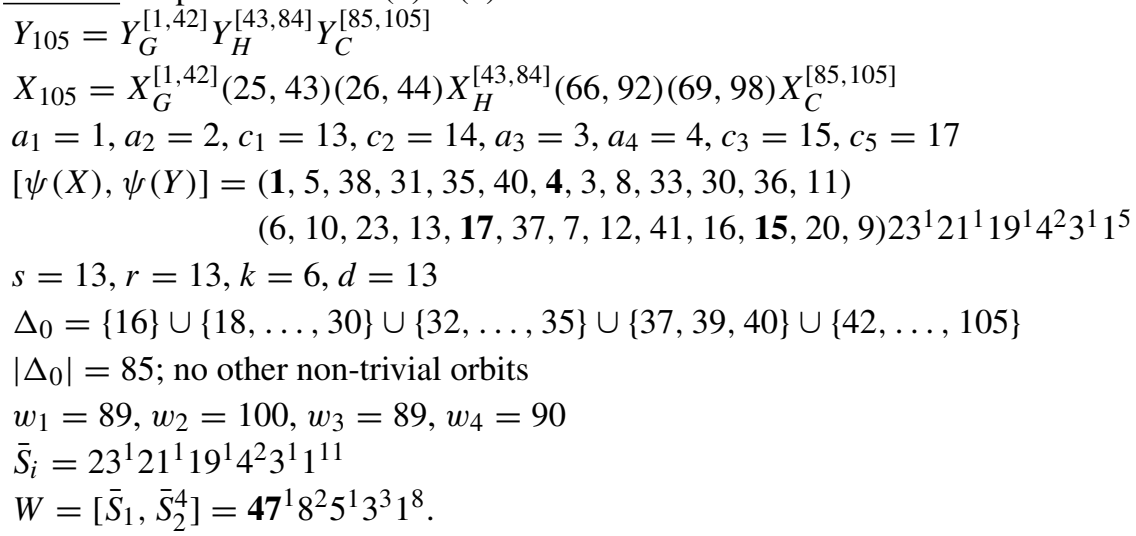


$n=100$. Representation $G(1) E(2) F$.

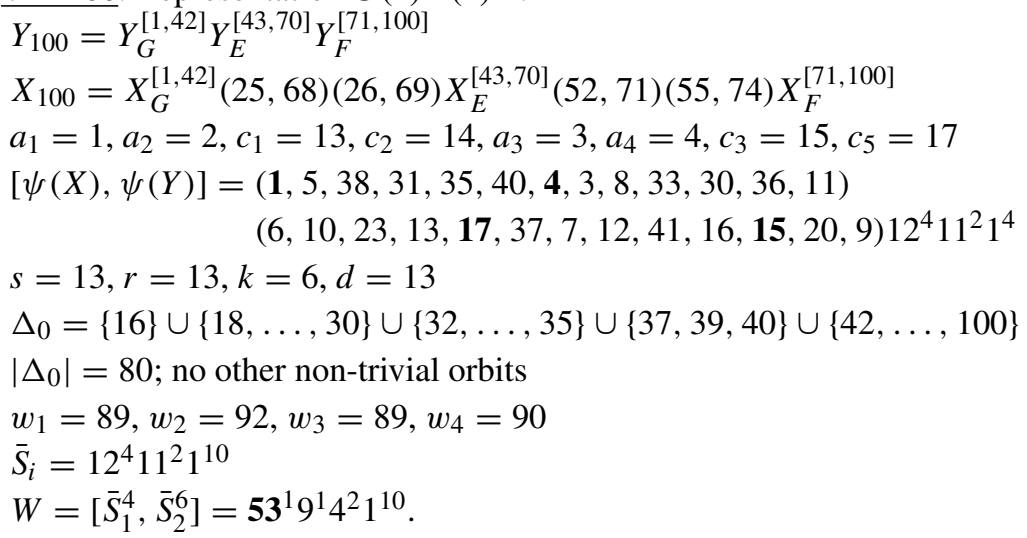

$\underline{n=98}$. Representation $G(1) E(2) E$.

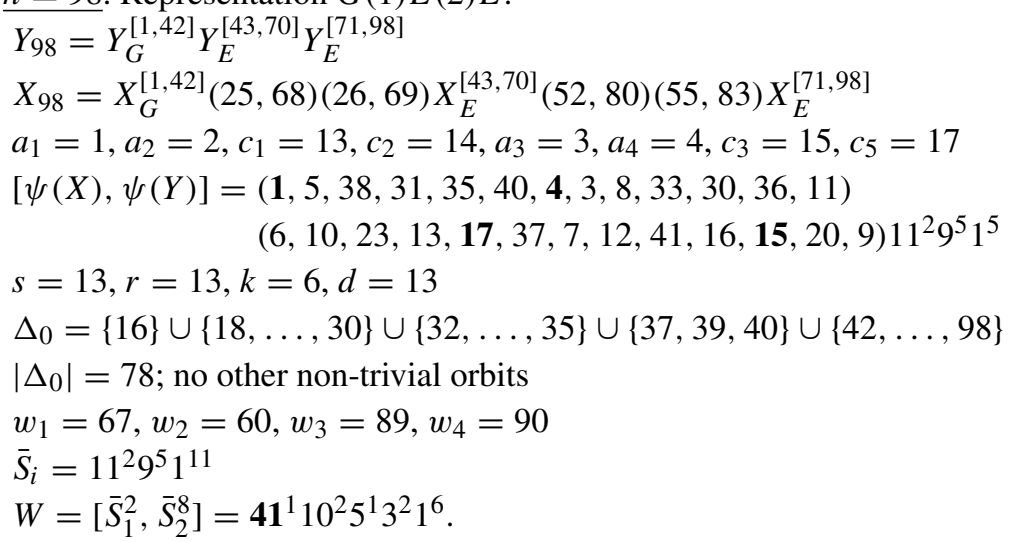

$\underline{n=93}$. Representation $G(1) Q(2) F$.

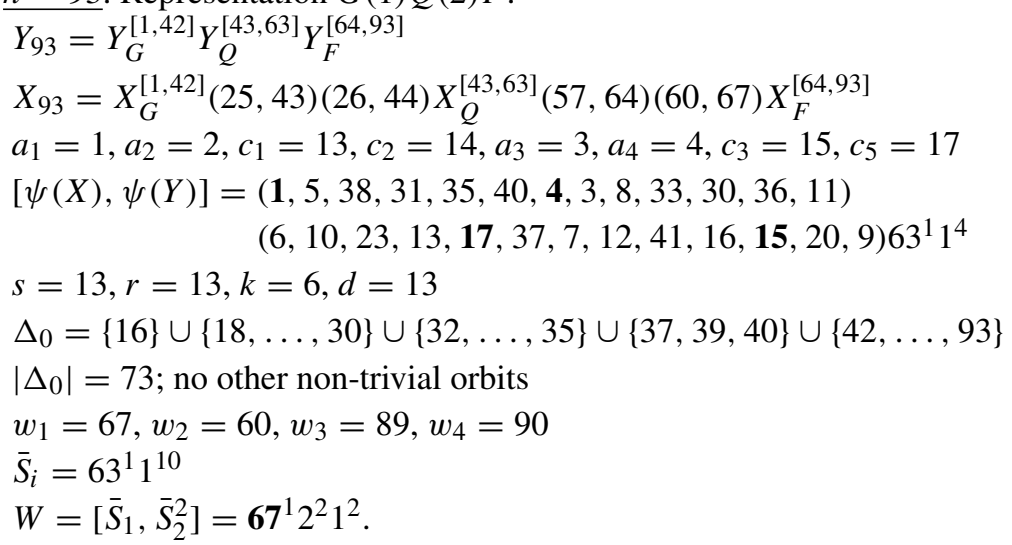


$\underline{n=92}$. Representation $G(1) E(2) D$.

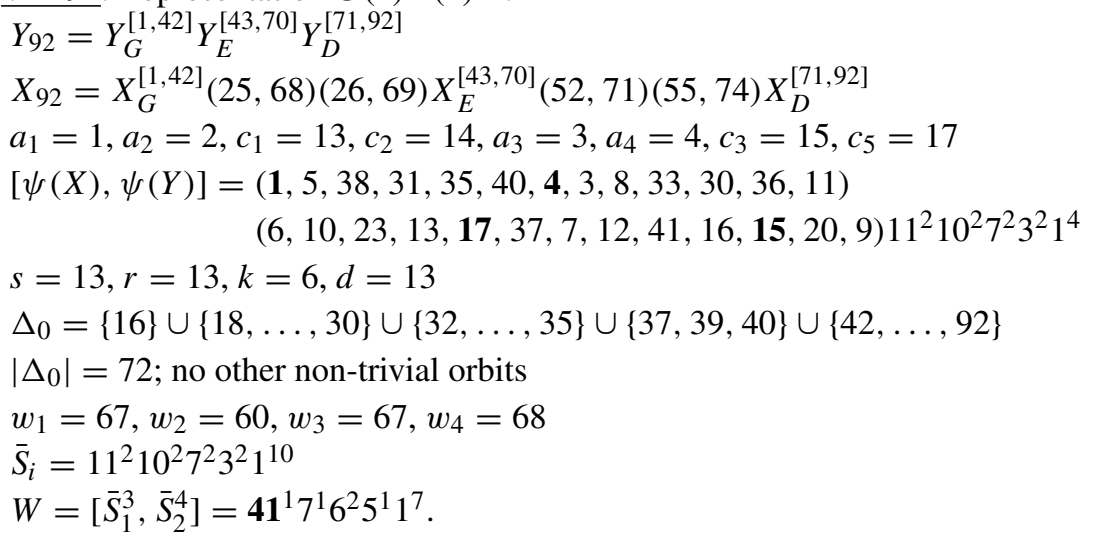

$\underline{n=91}$. Representation $G(1) G(1) O$.

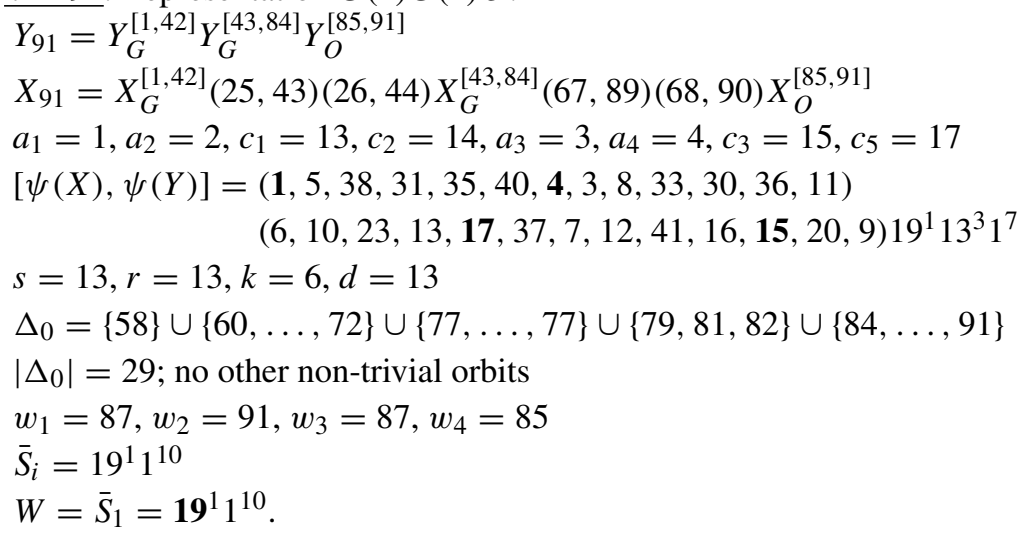

$\underline{n=85}$. Representation $G(1) E(2) B$.

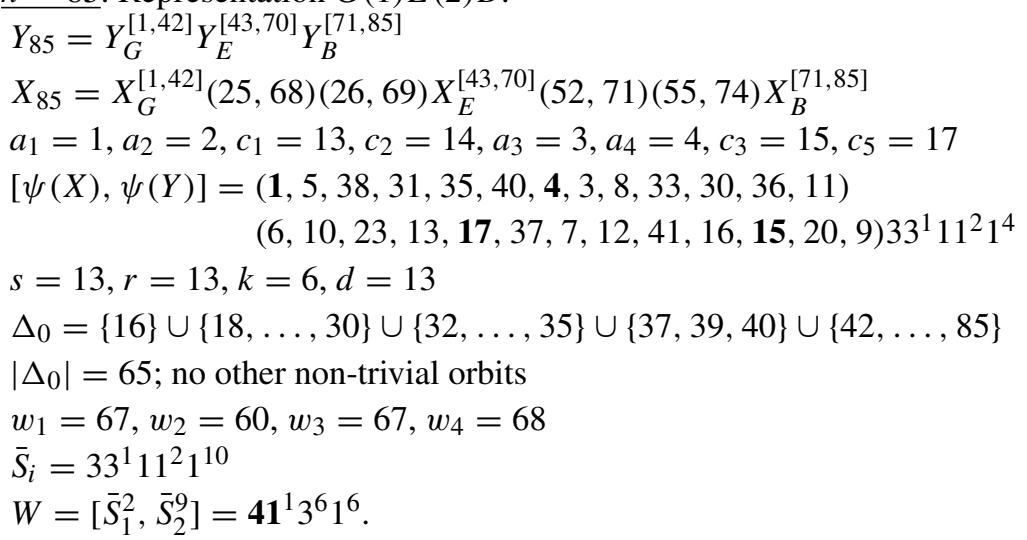


$\underline{n=77}$. Representation $G(1) E(2) O$.

$$
\begin{aligned}
& Y_{77}=Y_{G}^{[1,42]} Y_{E}^{[43,70]} Y_{O}^{[71,77]} \\
& X_{77}=X_{G}^{[1,42]}(25,68)(26,69) X_{E}^{[43,70]}(52,71)(55,75) X_{O}^{[71,77]} \\
& {[\psi(X), \psi(Y)]=(\mathbf{1}, 5,38,31,35,40, \mathbf{4}, 3,8,33,30,36,11)} \\
& s=13, r=13, k=6, d=13 \\
& \Delta_{0}=\{16\} \cup\{18, \ldots, 30\} \cup\{32, \ldots, 35\} \cup\{37,39,40\} \cup\{42, \ldots, 77\} \\
& \left|\Delta_{0}\right|=57 \text {; no other non-trivial orbits } \\
& w_{1}=53, w_{2}=57, w_{3}=53, w_{4}=54 \\
& \bar{S}_{i}=25^{1} 11^{2} 1^{10} \\
& W=\left[\bar{S}_{1}, \bar{S}_{2}^{2}\right]=\mathbf{4 3}^{1} 5^{1} 1^{9} \text {. }
\end{aligned}
$$$$
a_{1}=1, a_{2}=2, c_{1}=13, c_{2}=14, a_{3}=3, a_{4}=4, c_{3}=15, c_{5}=17
$$$$
(6,10,23,13,17,37,7,12,41,16,15,20,9) 25^{1} 11^{2} 1^{4}
$$

$\underline{n=70}$. Representation $G(1) E$.

$$
\begin{aligned}
& Y_{70}=Y_{G}^{[1,42]} Y_{E}^{[43,70]} \\
& X_{70}=X_{G}^{[1,42]}(25,68)(26,69) X_{E}^{[43,70]} \\
& {[\psi(X), \psi(Y)]=(\mathbf{1}, 5,38,31,35,40, \mathbf{4}, 3,8,33,30,36,11)} \\
& s=13, r=13, k=6, d=13 \\
& \Delta_{0}=\{16\} \cup\{18, \ldots, 30\} \cup\{32, \ldots, 35\} \cup\{37,39,40\} \cup\{42, \ldots, 70\} \\
& \left|\Delta_{0}\right|=50 \text {; no other non-trivial orbits } \\
& w_{1}=43, w_{2}=70, w_{3}=53, w_{4}=54 \\
& \bar{S}_{i}=11^{2} 9^{2} 1^{10} \\
& W=\left[\bar{S}_{1}, \bar{S}_{2}^{8}\right]=\mathbf{3 1}^{1} 11^{1} 1^{8} \text {. }
\end{aligned}
$$$$
a_{1}=1, a_{2}=2, c_{1}=13, c_{2}=14, a_{3}=3, a_{4}=4, c_{3}=15, c_{5}=17
$$$$
(6,10,23,13, \mathbf{1 7}, 37,7,12,41,16,15,20,9) 11^{2} 9^{2} 1^{4}
$$

$n=64$. Representation $G(1) R$.

$$
\begin{aligned}
& Y_{64}=Y_{G}^{[1,42]} Y_{R}^{[43,64]} \\
& X_{64}=X_{G}^{[1,42]}(25,43)(26,44) X_{R}^{[43,64]} \\
& {[\psi(X), \psi(Y)]=(\mathbf{1}, 5,38,31,35,40, \mathbf{4}, 3,8,33,30,36,11)} \\
& s=13, r=13, k=6, d=13 \\
& \Delta_{0}=\{16\} \cup\{18, \ldots, 30\} \cup\{32, \ldots, 35\} \cup\{37,39,40\} \cup\{42, \ldots, 64\} \\
& \left|\Delta_{0}\right|=44 \text {; no other non-trivial orbits } \\
& w_{1}=18, w_{2}=19, w_{3}=43, w_{4}=44 \\
& \bar{S}_{i}=17^{2} 1^{10} \\
& W=\left[\bar{S}_{1}, \bar{S}_{2}^{7}\right]=\mathbf{2 3}^{1} 8^{2} 3^{1} 1^{2} \text {. }
\end{aligned}
$$$$
a_{1}=1, a_{2}=2, c_{1}=13, c_{2}=14, a_{3}=3, a_{4}=4, c_{3}=15, c_{5}=17
$$$$
(6,10,23,13,17,37,7,12,41,16,15,20,9) 17^{2} 1^{4}
$$ 
$\underline{n=63}$. Representation $G(1) C$.

$Y_{63}=Y_{G}^{[1,42]} Y_{C}^{[43,63]}$
$X_{63}=X_{G}^{[1,42]}(25,43)(26,44) X_{C}^{[43,63]}$

$a_{1}=1, a_{2}=2, c_{1}=13, c_{2}=14, a_{3}=3, a_{4}=4, c_{3}=15, c_{5}=17$

$[\psi(X), \psi(Y)]=(\mathbf{1}, 5,38,31,35,40, \mathbf{4}, 3,8,33,30,36,11)$

$(6,10,23,13,17,37,7,12,41,16,15,20,9) 21^{1} 4^{2} 2^{2} 1^{4}$

$s=13, r=13, k=6, d=13$

$\Delta_{0}=\{16\} \cup\{18, \ldots, 30\} \cup\{32, \ldots, 35\} \cup\{37,39,40\} \cup\{42, \ldots, 63\}$

$\left|\Delta_{0}\right|=43$; no other non-trivial orbits

$w_{1}=39, w_{2}=22, w_{3}=43, w_{4}=44$

$\bar{S}_{i}=21^{1} 4^{2} 2^{2} 1^{10}$

$W=\left[\bar{S}_{1}^{3}, \bar{S}_{2}^{7}\right]=\mathbf{2 9}^{1} 5^{1} 1^{9}$.

$\underline{n=57}$. Representation $G(1) P$.

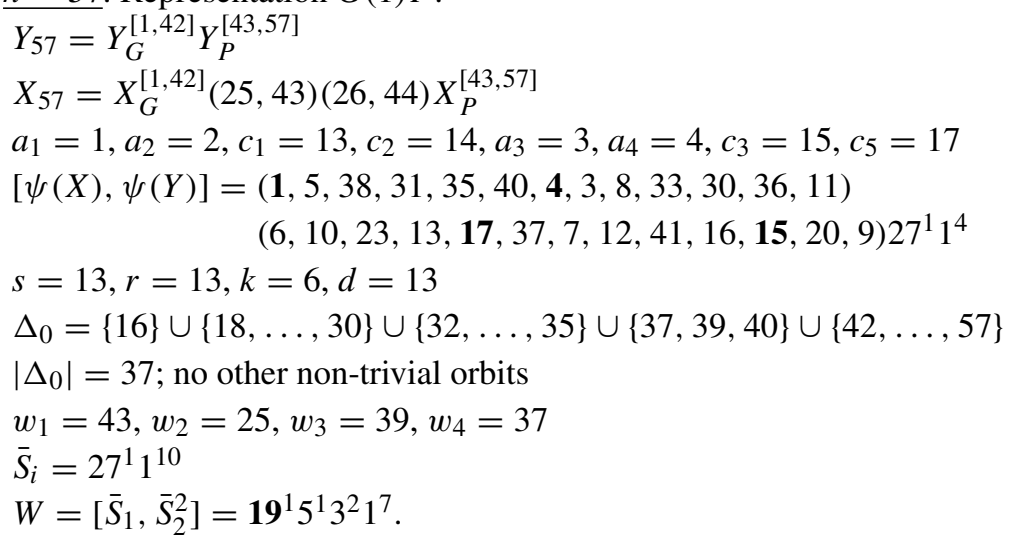

$\underline{n=49}$. Representation $G(1) O$.

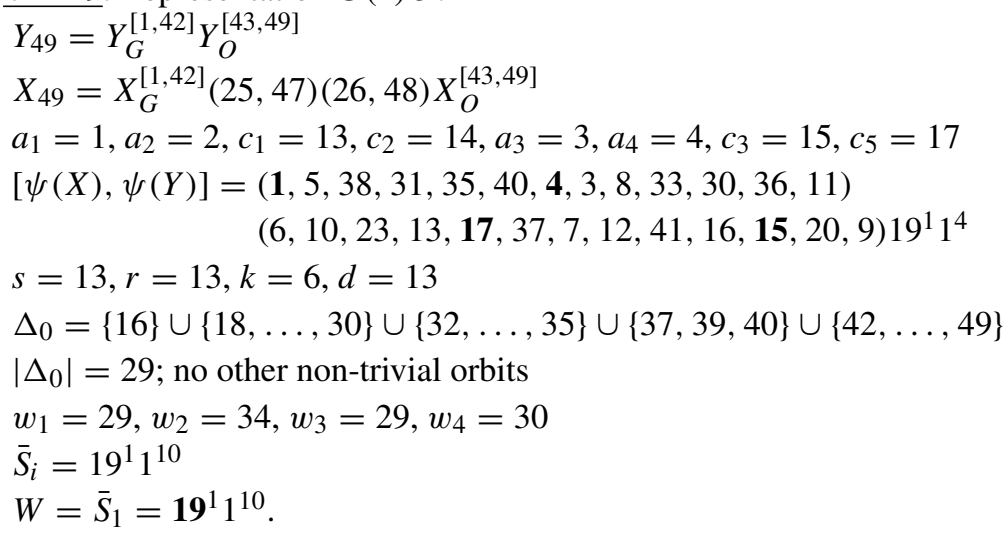


Appendix C.

This appendix contains the collection of MAGMA libraries that were used to obtain the information provided in Appendix B. These, as well as a "README" file, can be found at

$$
\text { http://www.lms.ac.uk/jcm/7/lms2004-042/appendix-c. }
$$

Acknowledgement The author is grateful to M. C. Tamburini for very useful discussions and comments.

\section{References}

1. M. D. E. CONDER, 'Generators for alternating and symmetric groups', J. London Math. Soc. (2) 22 (1980) 75-86. 302, 311

2. L. Di Martino and M. C. TAMburini, 'On the (2,3,7)-generation of maximal parabolic subgroups', J. Austral. Math. Soc. 71 (2001) 187-199. 303

3. L. Di Martino, M. C. Tamburini and A. E. Zalesski, 'On Hurwitz groups of low rank', Comm. Algebra 28 (2000) 5383-5404. 300

4. A. J. HaHn and O. T. O'MeARA, The classical groups and $K$-theory (Springer, 1989). 307

5. C. Jordan, 'Sur la limite de transivité des groupes non alternés', Bull. Soc. Math. France 1 (1873) 40-71. 312

6. A. LuCCHINI and M. C. TAMBURINI, 'Classical groups of large rank as Hurwitz groups', J. Algebra 219 (1999) 531-546. 300, 302, 303, 307

7. A. LuCchini, M. C. TAMburini and J. S. Wilson, 'Hurwitz groups of large rank', J. London Math. Soc. (2) 61 (2000) 81-92. 300, 301, 302, 303, 307, 310

8. L. L. Scотт, 'Matrices and cohomology', Ann. of Math. 105 (1977) 473-492. 313

9. W. W. Stothers, 'Subgroups of the (2,3,7)-triangle group', Manuscripta Math. 20 (1977) 323-334. 302, 311

10. M. C. TAmburini and M. Vsemirnov, 'Hurwitz groups and Hurwitz generation', Handbook of Algebra vol. 4, to appear. 300

11. H. WiElandt, Finite permutation groups (Academic Press, 1964). 312

M. Vsemirnov m.vsemirnovedpmms.cam.ac.uk, vsemir@pdmi.ras.ru http://logic.pdmi.ras.ru/ vsemir

Sidney Sussex College,

Sidney Street

Cambridge CB2 3HU

United Kingdom 